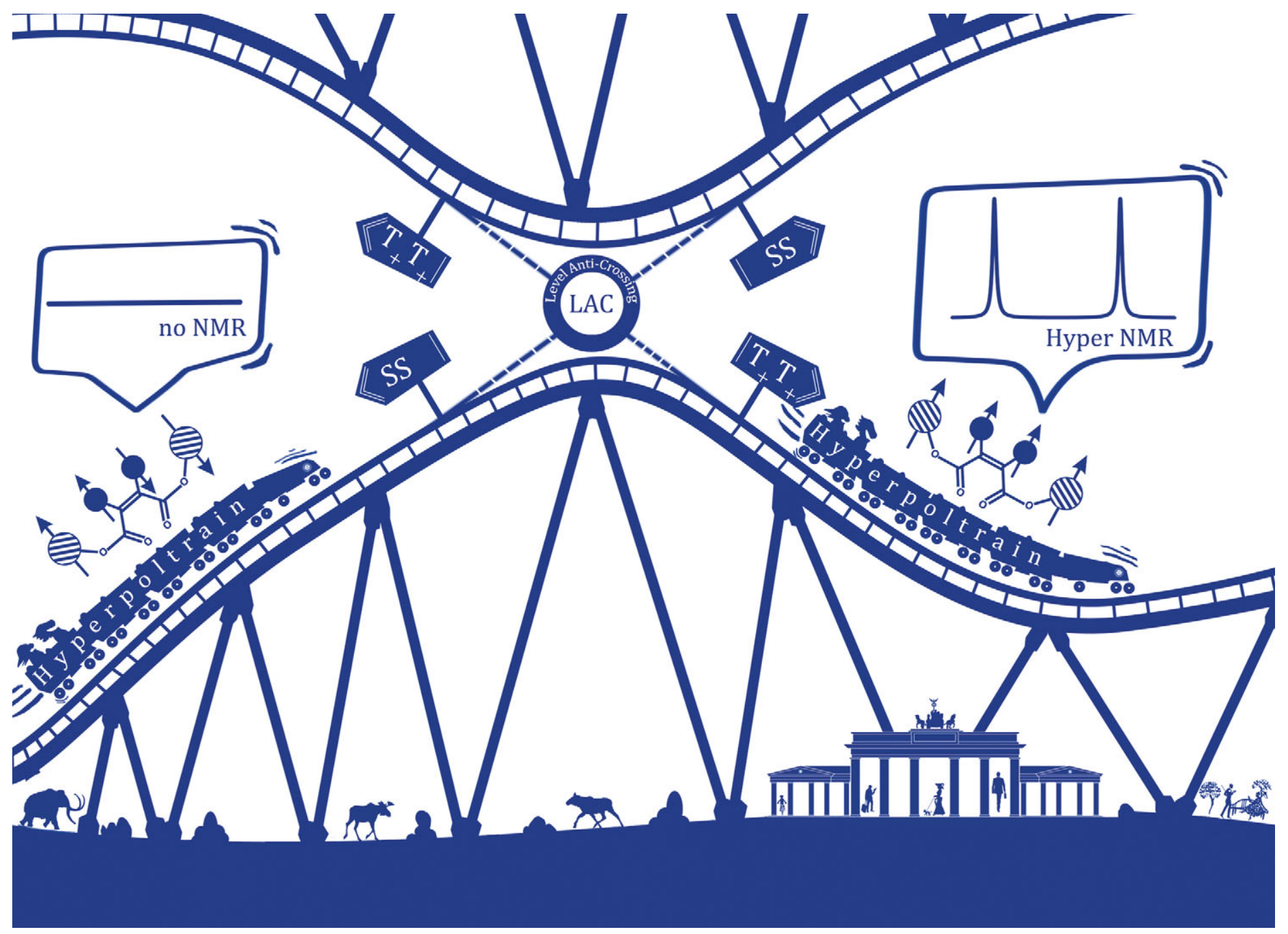

Showcasing research from cooperation between the International Tomography Center, Siberian Branch of the Russian Academy of Science, Russia (Andrey N. Pravdivtsev, Prof. Alexandra V. Yurkovskaya, Prof. Nikita N. Lukzen and Dr Konstantin Ivanov) and Free University of Berlin, Germany (Prof. Hans-Martin Vieth).

Title: Exploiting level anti-crossings (LACs) in the rotating frame for transferring spin hyperpolarization

Quantum-mechanical mixing at energy Level Anti-Crossing (LAC) regions is an efficient tool for manipulating nuclear spin hyperpolarization. The article demonstrates that LAC conditions in multispin systems can be fulfilled by applying a resonant RF-field with properly set frequency and amplitude. Controllable variation of these two parameters provides a new resource for efficient transfer of non-thermal spin order, for instance, for shifting proton order created by parahydrogen induced polarization, to target nuclei of choice.

As featured in:

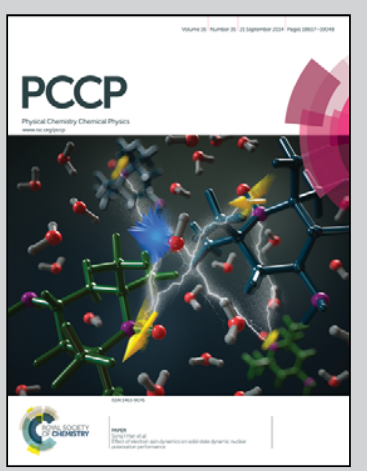

See Konstantin L. Ivanov et al., Phys. Chem. Chem. Phys., 2014, 16, 18707. 
Cite this: Phys. Chem. Chem. Phys., 2014, 16, 18707

Received 3rd April 2014 Accepted 16th May 2014

DOI: $10.1039 / c 4 c p 01445 f$

www.rsc.org/pccp

\title{
Exploiting level anti-crossings (LACs) in the rotating frame for transferring spin hyperpolarization
}

\author{
Andrey N. Pravdivtsev, ${ }^{a b}$ Alexandra V. Yurkovskaya, ${ }^{a b}$ Nikita N. Lukzen, ${ }^{a b}$ \\ Hans-Martin Vieth ${ }^{c}$ and Konstantin L. Ivanov*ab
}

\begin{abstract}
A method of transferring hyperpolarization among scalar-coupled nuclear spins is proposed, which is based on spin mixing at energy Level Anti-Crossing (LAC) regions. To fulfill LAC conditions a resonant RF-field was applied with properly set frequency and amplitude. In this situation LACs occur between the nuclear spin levels in the rotating doubly tilted reference frame. The validity of the approach is demonstrated by taking as an example the transfer of para-hydrogen induced polarization in a symmetric molecule, whose coupled spin network can be modeled as a four-spin $A A^{\prime} M M^{\prime}$-system with two pairs of 'isochronous' spins. For this spin system LAC positions have been identified; rules for the sign of spin polarization have been established. The dependence of the polarization transfer efficiency on the RF-field parameters and on the time profile of switching off the RF-field has been studied in detail; experimental results are in excellent agreement with the theory developed. In general, exploiting LACs in the rotating doubly tilted frame is a powerful tool for manipulating hyperpolarization in multispin systems.
\end{abstract}

\section{Introduction}

Hyperpolarizing nuclear spins is a strategy to increase the sensitivity of Nuclear Magnetic Resonance (NMR), which is often notoriously low because of poor Boltzmann population differences of spin states under equilibrium conditions. Spin systems, which are shifted far off thermal equilibrium, become hyperpolarized and exhibit intense NMR signals. Hyperpolarization techniques, such as Dynamic Nuclear Polarization (DNP), ${ }^{1-3}$ Optical Nuclear Polarization (ONP), ${ }^{4,5}$ Chemically Induced Dynamic Nuclear Polarization (CIDNP), ${ }^{6}$ optical pumping $(\mathrm{OP})^{7}$ of noble gas nuclei, Para-Hydrogen Induced Polarization (PHIP) $)^{8,9}$ and the Haupt effect, ${ }^{10}$ provide significant NMR enhancements and enable new promising applications. ${ }^{11-26}$

In hyperpolarization experiments polarization transfer among spins is often a crucial step, in particular, in the case of PHIP where initially only two protons originating from parahydrogen acquire non-thermal spin order. Optimization of polarization transfer is even more important for the SABRE (Signal Amplification By Reversible Exchange) technique, ${ }^{27}$ which is a new version of PHIP. In contrast to PHIP where a hydrogenation reaction leads to hyperpolarization, in the

\footnotetext{
${ }^{a}$ International Tomography Center, Siberian Branch of the Russian Academy of Science, Institutskaya 3a, Novosibirsk, 630090, Russia.

E-mail: ivanov@tomo.nsc.ru; Fax:+7(383)333-1399; Tel: +7(383)330-8868

${ }^{b}$ Novosibirsk State University, Pirogova 2, Novosibirsk, 630090, Russia

${ }^{c}$ Institut für Experimentalphysik, Freie Universität Berlin, Arnimallee 14, Berlin, 14195, Germany
}

SABRE case only a temporary $\mathrm{H}_{2}$-catalyst-ligand complex is formed and spin order is transferred from the para- $\mathrm{H}_{2}$ molecule to the ligand during the lifetime of this transient complex. Thus, an efficient polarization transfer step is a prerequisite for observing hyperpolarization-enhanced NMR signals of ligands.

To improve the polarization transfer efficiency it is advantageous to rely on coherent mechanisms rather than on cross-relaxation. Coherent polarization transfer mechanisms are operative ${ }^{28,29}$ at low magnetic fields where the spins are strongly coupled meaning that the difference in their Zeeman interactions with the field is larger than or comparable to their spin-spin coupling. The polarization transfer efficiency can be increased further ${ }^{30-37}$ by bringing the spin system to Level Anti-Crossing (LAC) regions, a way that also enables selective polarization transfer between well-defined spin orders. However, going to low fields represents a technical problem as it requires fast field-cycling. Thus, it is desirable to achieve strong coupling of spins and go to LAC regions while keeping the sample at the high magnetic field of the NMR spectrometer. This objective can be achieved by using an RF-field with properly set frequency and amplitude, which provides the strong coupling of spins (spin locking). For describing this situation we use as is common the reference frame rotating with the carrier frequency. It allows us to go to LACs in the rotating frame and to exploit them for polarization transfer. Such ideas have already been exploited, for instance, in $\mathrm{ONP}^{38-40}$ where spin mixing in the rotating frame enabled highly efficient polarization of nuclear spins by polarization transfer from non-thermally polarized triplet molecules. Quite recently,,$^{31,35-37,41}$ it was proposed to utilize LACs in PHIP: it was shown that PHIP is 
efficiently transferred at LAC regions; the coherent nature of the transfer process has also been established. Franzoni et al. ${ }^{37}$ have also demonstrated that LAC conditions can be fulfilled at high field in the presence of an RF-field with properly chosen parameters: at such LAC regions transfer of spin order becomes operative. We further develop ideas of using LACs in the rotating frame for funneling hyperpolarization to a specific spin order of choice. We also study in detail how the polarization transfer effects depend on the strength and frequency of the RF-field and also on the way it is switched. Our recent studies ${ }^{42,43}$ have shown that using different schemes of switching off the RF-field allows one to change the spectral pattern in almost any desirable way. Combination of the two concepts, LACs in the rotating frame and controllable fading of the RF-field, is thus an important development of hyperpolarizationbased NMR techniques.

Hence, in this work we pursue the methodology of LACs in the rotating frame for polarization transfer and develop a theoretical treatment of the problem. We describe conditions for LACs in the rotating frame, discuss the polarization transfer efficiency provided by such LACs and consider effects of the speed of RF-field fading. The theoretical treatment is based on considering coupled spins in the so-called rotating doubly tilted frame (DTF). We will show that adjusting two parameters, RF-frequency and RF-amplitude, gives an additional degree of freedom as compared to field-cycling experiments, where only one parameter, the external magnetic field strength, is variable. Using spin-locking also allows one to achieve net polarization of an entire spin system that initially had only multiplet spin order, giving an additional advantage of the method. Our study can be of importance for CIDNP, PHIP and, notably, SABRE techniques. This is because the SABRE-derived polarization of ligands can only be formed under strong coupling conditions, which so far require fast field-cycling between the NMR field and low fields where the ligand is efficiently polarized. As shown recently, ${ }^{34}$ the SABRE-derived polarization is highest when the system is brought to an LAC region. Using RF-fields to drive the spin system to an LAC can make SABRE a high-field technique, which does not require field-cycling, being advantageous for NMR spectroscopy and imaging applications.

Here we study PHIP effects in the symmetric molecule of maleic acid dimethyl ester, which is formed upon hydrogenation of dimethyl acetylenedicarboxylate with para-hydrogen. In Chart 1 the reaction scheme and the assignment of protons in maleic acid dimethyl ester are shown. In PHIP experiments the vinyl protons ( $\mathrm{AA}^{\prime}$ in our notation) originate from para-hydrogen, while the methyl protons $\left(\mathrm{MM}^{\prime}\right.$ in our notation) can be polarized by spin

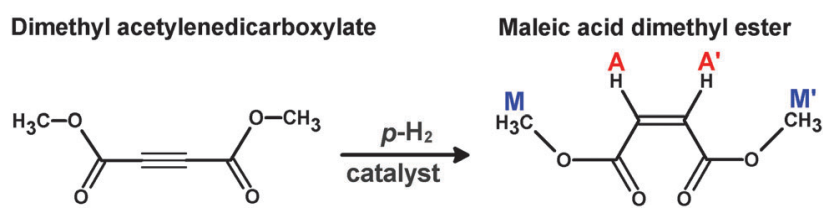

Chart 1 Scheme of hydrogenation of dimethyl acetylenedicarboxylate resulting in the formation of maleic acid dimethyl ester; assignment of $A^{\prime}$ (vinyl) and $\mathrm{MM}^{\prime}$ (methyl) protons is also given.
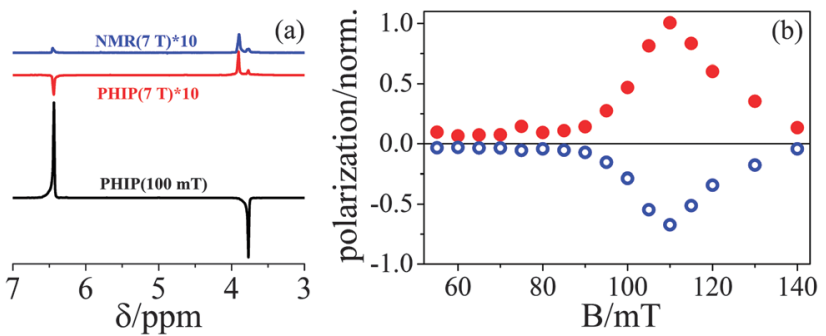

Fig. 1 PHIP spectra (a) and dependence of net polarization of the $A A^{\prime}$-protons (vinyl, full circles) and $M^{\prime} M^{\prime}$-protons (methyl, open circles) on the static external magnetic field (b). Experiments were done in the same way as described earlier; ${ }^{41}$ time of switching the field from the lowest position to the detection field was $0.5 \mathrm{~s}$. In (a) PHIP spectra are shown, which are taken for polarization fields of $100 \mathrm{mT}$ and $7 \mathrm{~T}$; for comparison we also show a $7 \mathrm{~T}$ thermal NMR spectrum.

mixing at LACs. As found out by Buljubasich et $a l^{35}$ the spin system of maleic acid dimethyl ester can be modeled in good approximation as a four-spin $\mathrm{AA}^{\prime} \mathrm{MM}^{\prime}$-system by considering each methyl group as a single proton. The $\mathrm{AA}^{\prime} \mathrm{MM}^{\prime}$-system is a system of two 'isochronous' $\mathrm{AA}^{\prime}$-spins with the same chemical shift, which are coupled to another pair, $\mathbf{M M}^{\prime}$, of 'isochronous' spins.

Importantly, PHIP in such a spin system leads to observable NMR signals only after spin mixing at LAC regions has occurred; otherwise the $\mathrm{AA}^{\prime}$-spins remain in their singlet state, which is NMR silent. This is demonstrated in Fig. 1: when hydrogenation is performed at high field the NMR line intensity is very small. When the field is set to $100 \mathrm{mT}$ PHIP appears resulting in strongly enhanced NMR lines of the vinyl and methyl protons, which are positive and negative, respectively. The line intensities in the PHIP spectrum are considerably larger than those in the thermal NMR spectrum, as also shown in Fig. 1. The magnetic field dependence demonstrates that PHIP is efficiently formed only in a certain field range, which is determined ${ }^{35}$ by the LAC regions in the spin system under study.

\section{Theory}

Before going to the quantum-mechanical description of the problem let us briefly introduce the LAC concept. ${ }^{44,45}$ An LAC occurs when two energy levels, $|m\rangle$ and $|n\rangle$, cross (i.e., have the same energy) but a coupling matrix element of the Hamiltonian, $V_{m n}=\langle m|\hat{H}| n\rangle$, splits them. As a result, a level crossing (LC) turns into an avoided crossing also termed Level-Anti-Crossing (LAC). Thus, an LAC is a combination of two ingredients: (i) matching (energies of two states coincide) and (ii) coupling (there is a matrix element, which mixes the two states). At the LAC region the initial states, $|m\rangle$ and $|n\rangle$, become 'entangled': the eigenstates of the Hamiltonian are given by a mixture of $|m\rangle$ and $|n\rangle$. For this reason population can be efficiently transferred between the two states when the LAC conditions are fulfilled. Therefore, the LAC concept is crucial for our work. It is possible to go to LC points by tuning the external magnetic field; however, LACs can also be accessed in the rotating frame at high field. 
In this case they work in a similar way enabling population exchange between the crossing levels and, thereby, polarization transfer in the spin system.

It is important to mention that LACs in the rotating frame are often exploited in NMR; the most well-known example is given by the Hartmann-Hahn cross-polarization experiment. ${ }^{46}$ In this case polarization is transferred between heteronuclei when the matching conditions are fulfilled so that there is an LC in the rotating frame. In the presence of coupling (scalar coupling in liquids and dipolar coupling in solids) the LC turns into an LAC and populations of the crossing levels are exchanged and polarization is transferred between the nuclear spins. In the case under study we use LACs in the rotating frame to transfer PHIP among groups of spins.

Let us start from a theoretical description of the problem. First, to give a clear idea of how the method works, we derive analytical results for a four-spin system of the type $\mathrm{AA}^{\prime} \mathrm{MM}^{\prime}$. We assume that the system has a $C_{\mathrm{s}}$-symmetry, which is reflected by two pairs of identical scalar couplings among the spins: $J_{\mathrm{AM}}=J_{\mathrm{A}^{\prime} \mathbf{M}^{\prime}}$ and $J_{\mathrm{AM}^{\prime}}=J_{\mathrm{A}^{\prime} \mathrm{M}}$. We have chosen such a system because it corresponds to one that has been experimentally studied. Previously Buljubasich et al. ${ }^{35}$ have investigated PHIP effects in such a system and demonstrated the importance of LACs for the observed polarization. This system is known to have several LACs at distinct external magnetic fields; the LAC positions can be found analytically. ${ }^{35}$ Recently the same group has found that LAC conditions in the $\mathrm{AA}^{\prime} \mathrm{MM}^{\prime}$-system can be fulfilled not only when the permanent magnetic field is varied but also when at a constant static field the RF-field strength is properly set. ${ }^{37}$ Spin mixing at such LAC regions was found and exploited to convert the initial singlet spin order of parahydrogen into enhanced NMR signals. ${ }^{37}$ An analytical treatment is also possible for a few other types of spin systems; ${ }^{34}$ however, here, for the sake of clarity, we consider only the $\mathrm{AA}^{\prime} \mathrm{MM}^{\prime}$-system. This is done, on the one hand, to demonstrate that for PHIP transfer more than two spins are required; on the other hand, the spin system should not be too complex to enable analytical treatment of the problem.

Here we study hyperpolarization (in our case, PHIP) transfer effects in the same kind of system, though in greater detail. In the theoretical section we discuss conditions for having LACs in the general case, i.e., at arbitrary frequency and strength of the RF-field. To do this, we consider the Hamiltonian of the spin system in the DTF (vide infra). We find LAC positions and identify the levels, which cross in the DTF, and find out, to which levels in the non-tilted rotating frame they correspond. In this section we also discuss how different regimes of switching off the external RF field affect the final polarization pattern, which is observed by NMR spectroscopy.

Hereafter we assume that the $\mathrm{AA}^{\prime}$-spins are prepared in their singlet state, whereas the $\mathrm{MM}^{\prime}$-spins have zero initial polarization. Such a spin order gives no NMR signal: enhanced NMR lines in this case can only be formed due to spin mixing at LAC regions. ${ }^{34,35,37}$ We are thus interested only in LACs involving the singlet state of the $\mathrm{AA}^{\prime}$-spins. When an oscillating RF-field is applied to the spin system its Hamiltonian in the rotating frame of reference takes the form (in frequency units):

$$
\begin{aligned}
\hat{H}_{\mathrm{rf}}= & -\left(\nu_{\mathrm{A}}-\nu_{\mathrm{rf}}\right)\left(\hat{I}_{\mathrm{A} z}+\hat{I}_{\mathrm{A}^{\prime} z}\right)-\left(\nu_{\mathrm{M}}-\nu_{\mathrm{rf}}\right)\left(\hat{I}_{\mathrm{M} z}+\hat{I}_{\mathrm{M}^{\prime} z}\right) \\
& -\nu_{1}\left(\hat{I}_{\mathrm{A} x}+\hat{I}_{\mathrm{A}^{\prime} x}+\hat{I}_{\mathrm{M} x}+\hat{I}_{\mathrm{M}^{\prime} x}\right)+\hat{H}_{\mathrm{J}}
\end{aligned}
$$

where the part, $\hat{H}_{\mathrm{J}}$, describing the $J$-couplings is as follows:

$$
\begin{aligned}
\hat{H}_{\mathbf{J}}= & J_{\mathrm{AA}^{\prime}}\left(\hat{\mathbf{I}}_{\mathbf{A}} \cdot \hat{\mathbf{I}}_{\mathbf{A}^{\prime}}\right)+J_{\mathbf{M M}^{\prime}}\left(\hat{\mathbf{I}}_{\mathbf{M}^{\prime}} \cdot \hat{\mathbf{I}}_{\mathbf{M}^{\prime}}\right)+J_{\mathrm{AM}}\left\{\left(\hat{\mathbf{I}}_{\mathbf{A}} \cdot \hat{\mathbf{I}}_{\mathbf{M}}\right)+\left(\hat{\mathbf{I}}_{\mathrm{A}^{\prime}} \cdot \hat{\mathbf{I}}_{\mathbf{M}^{\prime}}\right)\right\} \\
& +J_{\mathrm{AM}^{\prime}}\left\{\left(\hat{\mathbf{I}}_{\mathbf{A}} \cdot \hat{\mathbf{I}}_{\mathbf{M}^{\prime}}\right)+\left(\hat{\mathbf{I}}_{\mathbf{A}^{\prime}} \cdot \hat{\mathbf{I}}_{\mathbf{M}}\right)\right\}
\end{aligned}
$$

Here we introduced $\nu_{\mathrm{rf}}$ as the frequency of the RF field and $\nu_{1}$ as its amplitude. An exact solution of the eigen-problem of such a Hamiltonian is, generally, not feasible; however, it is possible to develop approximate solutions. To do this following Buljubasich et $a l .{ }^{35}$ we split the Hamiltonian $\hat{H}_{\text {rf }}$ in the main part

$$
\begin{aligned}
\hat{H}_{0}= & -\left(\nu_{\mathrm{A}}-\nu_{\mathrm{rf}}\right)\left(\hat{I}_{\mathrm{A} z}+\hat{I}_{\mathrm{A}^{\prime} z}\right)-\left(\nu_{\mathrm{M}}-\nu_{\mathrm{rf}}\right)\left(\hat{I}_{\mathrm{M} z}+\hat{I}_{\mathrm{M}^{\prime} z}\right) \\
& -\nu_{1}\left(\hat{I}_{\mathrm{A} x}+\hat{I}_{\mathrm{A}^{\prime} x}+\hat{I}_{\mathrm{M} x}+\hat{I}_{\mathrm{M}^{\prime} x}\right)+\hat{H}_{\mathrm{J} 0}
\end{aligned}
$$

and the perturbation

$$
\hat{V}=J_{\mathrm{AM}}\left\{\left(\hat{\mathbf{I}}_{\mathrm{A}} \cdot \hat{\mathbf{I}}_{\mathbf{M}}\right)+\left(\hat{\mathbf{I}}_{\mathrm{A}^{\prime}} \cdot \hat{\mathbf{I}}_{\mathbf{M}^{\prime}}\right)\right\}+J_{\mathrm{AM}^{\prime}}\left\{\left(\hat{\mathbf{I}}_{\mathbf{A}} \cdot \hat{\mathbf{I}}_{\mathbf{M}^{\prime}}\right)+\left(\hat{\mathbf{I}}_{\mathbf{A}^{\prime}} \cdot \hat{\mathbf{I}}_{\mathbf{M}}\right)\right\}
$$

Here we introduced

$$
\hat{H}_{\mathrm{J} 0}=J_{\mathrm{AA}^{\prime}}\left(\hat{\mathbf{I}}_{\mathrm{A}} \cdot \hat{\mathbf{I}}_{\mathrm{A}^{\prime}}\right)+J_{\mathrm{MM}^{\prime}}\left(\hat{\mathbf{I}}_{\mathbf{M}} \cdot \hat{\mathbf{I}}_{\mathrm{M}^{\prime}}\right)
$$

The Hamiltonian $\hat{H}_{0}$ does not change the total spin of the $\mathrm{AA}^{\prime}$ - and $\mathrm{MM}^{\prime}$-spins; thus it does not induce singlet-triplet transitions in each group of spins and gives no polarization transfer. Polarization transfer effects are conditioned merely by the perturbation, $\hat{V}$.

\section{Rotating doubly tilted frame of reference}

To solve the eigen-problem of $\hat{H}_{0}$ we use the following method. The spins are quantized not along the $z$-axis (direction of the NMR field) but along their effective fields in the rotating frame (see Fig. 2). For the $\mathrm{AA}^{\prime}$-spins the field vector is

$$
B_{\mathrm{A}}=\left(B_{1}, 0,-2 \pi\left(\nu_{\mathrm{A}}-\nu_{\mathrm{rf}}\right) / \gamma\right)
$$

for the $\mathrm{MM}^{\prime}$-spins it is

$$
B_{\mathrm{M}}=\left(B_{1}, 0,-2 \pi\left(\nu_{\mathrm{M}}-\nu_{\mathrm{rf}}\right) / \gamma\right)
$$
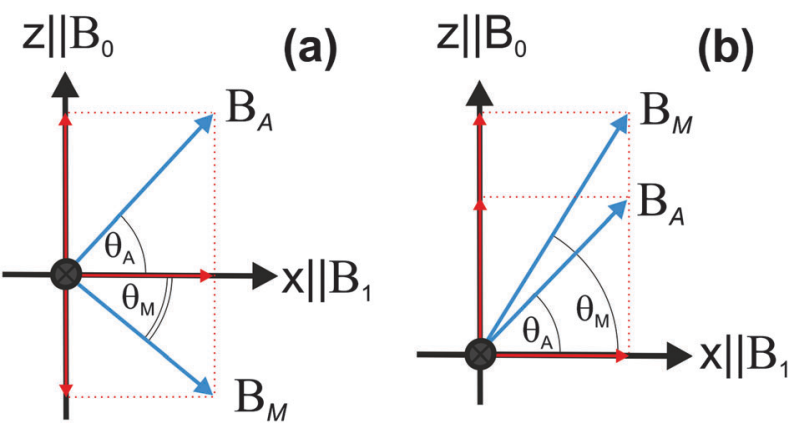

Fig. 2 Effective fields in the rotating doubly tilted frame; $z$-axis is parallel to the $B_{0}$-field, $x$-axis is parallel to the $B_{1}$-field. Here $\nu_{\mathrm{M}}>\nu_{\mathrm{rf}}>\nu_{\mathrm{A}}$ (subplot a) and $\nu_{\mathrm{M}}>\nu_{\mathrm{A}}>\nu_{\mathrm{rf}}$ (subplot $\mathrm{b}$ ). The inclination angles for the effective fields are denoted as $\theta_{\mathrm{A}}$ and $\theta_{\mathrm{M}}$. 
Here $\gamma$ is the nuclear gyromagnetic ratio; $B_{1}=2 \pi \nu_{1} / \gamma$ is the RF-field strength. Generally, both fields have different directions in the $x, z$-plane, such frames of reference are termed 'rotating doubly tilted' frames, DTF. ${ }^{47,48}$ In addition, the strength of the two effective fields is, in general, different except for the case where $\nu_{\mathrm{rf}}=\left(\nu_{\mathrm{A}}+\nu_{\mathrm{M}}\right) / 2$. The direction and strength of the two field vectors, $B_{\mathrm{A}}$ and $B_{\mathrm{M}}$, tend to coincide when $\nu_{1} \gg\left|\nu_{\mathrm{A}}-\nu_{\mathrm{rf}}\right|,\left|\nu_{\mathrm{M}}-\nu_{\mathrm{rf}}\right|$. Hereafter in the analytical treatment the $\mathrm{AA}^{\prime}$-spins are quantized along $B_{\mathrm{A}}$, whilst the $\mathrm{MM}^{\prime}$ spins are quantized along a different axis, $B_{\mathrm{M}}$. In both pairs of spins the states are written in the singlet-triplet basis.

In the DTF the spin Hamiltonian takes the form:

$$
\hat{H}_{\mathrm{dtf}}=-\tilde{\nu}_{\mathrm{A}}\left(\hat{I}_{\mathrm{A} z}+\hat{I}_{\mathrm{A}^{\prime} z}\right)-\tilde{\nu}_{\mathrm{M}}\left(\hat{I}_{\mathrm{Mz}}+\hat{I}_{\mathrm{M}^{\prime} z}\right)+\hat{H}_{\mathrm{Jo}}+\hat{V}_{\mathrm{dtf}}
$$

Hereafter all operators and spin states and defined in the DTF are denoted by the subscript 'dtf'. The precession frequencies of the pairs of spins about their effective field axes are:

$$
\tilde{\nu}_{\mathrm{A}, \mathrm{M}}=\sqrt{\left(\nu_{1}\right)^{2}+\left(\nu_{\mathrm{A}, \mathrm{M}}-\nu_{\mathrm{rf}}\right)^{2}}
$$

The $\hat{H}_{\mathrm{J} 0}$ term does not change, while the perturbation has to be re-defined in the new frame of reference: $\hat{V} \rightarrow \hat{V}_{\mathrm{dtf}}$. We discuss below the relevant terms in the perturbation. Since the main part of the Hamiltonian $\hat{H}_{\text {dff }}$ is formally the same as that for the Hamiltonian in the static field, one can use the results from earlier studies ${ }^{34,35}$ to find the level crossing position. In the system considered there are two kinds of level crossings occurring when ${ }^{34,35,37}$

$$
\begin{aligned}
& \pm\left(\tilde{\nu}_{\mathrm{A}}-\tilde{\nu}_{\mathrm{M}}\right)=J_{\mathrm{AA}^{\prime}}-J_{\mathrm{MM}^{\prime}} \text { crossing of }\left|\mathrm{ST}_{ \pm}\right\rangle_{\mathrm{dtf}} \text { and }\left|\mathrm{T}_{ \pm} \mathrm{S}\right\rangle_{\mathrm{dtf}} \\
& \pm\left(\tilde{\nu}_{\mathrm{A}}+\tilde{\nu}_{\mathrm{M}}\right)=J_{\mathrm{AA}^{\prime}}+J_{\mathrm{MM}^{\prime}} \text { crossing of }|\mathrm{SS}\rangle_{\mathrm{dtf}} \text { and }\left|\mathrm{T}_{ \pm} \mathrm{T}_{\mp}\right\rangle_{\mathrm{dtf}}
\end{aligned}
$$

Here the ket-states are defined in the DTF; to the position of the second pair of LCs there is also an additional contribution coming from the small couplings, $J_{\mathrm{AM}}, J_{\mathrm{AM}^{\prime}}$, which we neglect here. When these conditions are fulfilled the first requirement for a LAC (matching) is met.

Before discussing different cases let us also define how different positions of the RF-frequency, $\nu_{\mathrm{rf}}$, with respect to the NMR lines are termed hereafter in the text. By the 'center of the NMR spectrum' we define the frequency position equal to the average of the two NMR frequencies, $\nu_{\mathrm{A}}$ and $\nu_{\mathrm{M}}$, i.e., $\nu_{\mathrm{rf}}=\langle\nu\rangle=$ $\left(\nu_{\mathrm{A}}+\nu_{\mathrm{M}}\right) / 2$. By RF-frequencies placed 'inside' the NMR spectrum we mean $\nu_{\mathrm{rf}}$ values such that $\nu_{\mathrm{A}}>\nu_{\mathrm{rf}}>\nu_{\mathrm{M}}$ or $\nu_{\mathrm{M}}>\nu_{\mathrm{rf}}>\nu_{\mathrm{A}}$ (the frequency position is between the two NMR lines). When $\nu_{\mathrm{rf}}>$ $\nu_{\mathrm{A}, \mathrm{M}}$ or $\nu_{\mathrm{A}, \mathrm{M}}>\nu_{\mathrm{rf}}$ (the frequency is either higher or lower than both NMR lines) the corresponding RF-frequency is placed 'outside' the NMR spectrum.

The two pairs of crossings defined in eqn (10) occur when the RF-frequency is located almost at the center of the spectrum being only slightly detuned from it: at the center of the spectrum $\tilde{\nu}_{\mathrm{A}}=\tilde{\nu}_{\mathbf{M}}$ it is thus necessary to step a little aside to have a frequency difference equal to $\pm\left(J_{\mathrm{AA}^{\prime}}+J_{\mathrm{MM}^{\prime}}\right)$ or $\pm\left(J_{\mathrm{AA}^{\prime}}-J_{\mathrm{MM}^{\prime}}\right)$. One should note that the level crossing conditions also depend on the $J_{\mathrm{AM}}$ and $J_{\mathrm{AM}^{\prime}}$ couplings; ${ }^{35}$ however, for the sake of simplicity we assume that these couplings are much smaller than $\left(J_{\mathrm{AA}^{\prime}} \pm J_{\mathrm{MM}^{\prime}}\right)$ and neglect them when calculating the level crossing positions. When needed, it is easy to take them into account as done previously. ${ }^{35}$

Now let us see whether the second condition, coupling, is also met, as it is needed to change a level crossing into an LAC. The coupling is given by the perturbation term $\hat{V}_{\text {dtf }}$. The task is now that we have to specify the scalar spin-spin interactions in the representation where the coupled spins are quantized along different axes, $B_{\mathrm{A}}$ and $B_{\mathrm{M}}$. Here we do not write down all contributions to $\hat{V}_{\mathrm{dtf}}$ but only the terms, which cause transitions between the crossing levels (and slightly change the position of LACs, which is, however, neglected here). Thus, it is necessary to keep only terms containing products of one rising spin operator for the $\mathrm{AA}^{\prime}$-spins and one lowering spin operator for the $\mathrm{MM}^{\prime}$-spins and vice versa. The result depends on the angle between the field vectors $B_{\mathrm{A}}$ and $B_{\mathrm{M}}$ (which are generally not parallel to each other). When the RF-frequency is placed 'inside' the NMR spectrum, this angle is equal to $\theta_{\mathrm{AM}}=$ $\theta_{\mathbf{A}}+\theta_{\mathbf{M}}=2 \Theta$. When the RF frequency is placed 'outside' the NMR spectrum then $2 \Theta=\theta_{\mathrm{AM}}=\theta_{\mathrm{A}}-\theta_{\mathrm{M}}$. Although in this situation LAC conditions can also be fulfilled it requires significantly higher values of $\nu_{1}$; therefore in most cases we deal with the situation where the RF-frequency is placed 'inside' the NMR spectrum. The angle $\Theta$ is expressed via the two angles, $\theta_{\mathrm{A}}$ and $\theta_{\mathrm{M}}$ (see Fig. 2), which give the orientation of the two $z$-axes of the DTF with respect to the permanent field direction. The term $\hat{V}_{\mathrm{dtf}}$ can be then written as follows:

$$
\begin{aligned}
& \tilde{V}_{\mathrm{dtf}}=\frac{\cos ^{2} \Theta}{2} J_{\mathrm{AM}}\left\{\left(\hat{I}_{\mathrm{A}_{+}} \hat{I}_{\mathrm{M}_{-}}+\hat{I}_{\mathrm{A}_{-}} \hat{I}_{\mathrm{M}_{+}}\right)+\left(\hat{I}_{\mathrm{A}_{+}^{\prime}} \hat{I}_{\mathrm{M}_{-}^{\prime}}+\hat{I}_{\mathrm{A}_{-}^{\prime}} \hat{I}_{\mathrm{M}_{+}^{\prime}}\right)\right\} \\
& +\frac{\cos ^{2} \Theta}{2} J_{\mathrm{A}^{\prime} \mathrm{M}}\left\{\left(\hat{I}_{\mathrm{A}_{+}} \hat{I}_{\mathrm{M}_{-}^{\prime}}+\hat{I}_{\mathrm{A}_{-}} \hat{I}_{\mathrm{M}_{+}^{\prime}}\right)+\left(\hat{I}_{\mathrm{A}_{+}^{\prime}} \hat{I}_{\mathrm{M}_{-}}+\hat{I}_{\mathrm{A}_{-}^{\prime}} \hat{I}_{\mathrm{M}_{+}}\right)\right\} \\
& + \text {additional terms }
\end{aligned}
$$

Here we do not specify the additional terms, since they do not induce flip-flop transitions, which are the only transitions capable of mixing the crossing levels. Thus, in the DTF the couplings between spins from different groups are multiplied by a coefficient $\alpha=\frac{\cos ^{2} \Theta}{2}$ for the flip-flop term. Taking results from previous studies ${ }^{35}$ we conclude that couplings between the $\mathrm{AA}^{\prime}$ - and $\mathrm{MM}^{\prime}$-groups turn all level crossings into LACs, unless $\alpha$ vanishes. This is the case when $\Theta \rightarrow \frac{\pi}{2}$, i.e., the two effective fields tend to be anti-parallel, which happens at very low RF-field amplitude as compared to the half-width of the NMR spectrum: $\left(\nu_{\mathrm{A}, \mathrm{M}}-\nu_{\mathrm{rf}}\right) \gg \nu_{1}$. Thus, to make the method operative and to form LACs it is necessary that the RF-field is sufficiently strong. Under these conditions level crossings, see eqn (10), turn into LACs and there is efficient spin mixing occurring at these LACs:

$$
\left|S T_{ \pm}\right\rangle_{\text {dtf }} \leftrightarrow\left|\mathrm{T}_{ \pm} \mathrm{S}\right\rangle_{\mathrm{dtf}} \text { or }|\mathrm{SS}\rangle_{\mathrm{dtf}} \leftrightarrow\left|\mathrm{T}_{ \pm} \mathrm{T}_{\bar{F}}\right\rangle_{\mathrm{dtf}}
$$

resulting in efficient spin order transfer between the $\mathrm{AA}^{\prime}$ - and $\mathrm{MM}^{\prime}$-spins. 
In this context also the individual inclination angles $\theta_{\mathrm{A}^{\prime}}, \theta_{\mathbf{M}}$ are of importance. For instance, when the $B_{\mathrm{A}}, B_{\mathrm{M}}$ vectors become parallel to the $x$-axis the flips of the $\mathbf{M M}^{\prime}$-spins cannot produce their net polarization along the $z$-axis. As a consequence, there is no $z$-polarization of the $\mathbf{M M}^{\prime}$-spins but only transverse polarization. In order to produce longitudinal polarization, the $z$-component of the $B_{\mathbf{M}}$ vector has to be considerably large, which is given by $\sin \theta_{\mathbf{M}}$. Similar considerations hold for the $\mathrm{AA}^{\prime}$ spins. Thus, not only the matching and coupling conditions have to be fulfilled but also the effective field in the DTF must be properly inclined. In some of the experiments the choice of axes of the DTF becomes important. We will address this point later in the text.

\section{LACs in the doubly tilted frame}

Let us now discuss what polarization patterns can be obtained for different LACs and what the precise conditions for the LC points are. Initially, only the states with singlet character with respect to the $\mathrm{AA}^{\prime}$-spins are populated. Spin mixing at the LAC regions leads to the following transitions:

$$
\begin{gathered}
\text { LAC1: }\left|\mathrm{ST} T_{+}\right\rangle_{\mathrm{dtf}} \rightarrow\left|\mathrm{T}_{+} \mathrm{S}\right\rangle_{\mathrm{dtf}} \text { at }\left(\tilde{\nu}_{\mathrm{A}}-\tilde{\nu}_{\mathrm{M}}\right)=J_{\mathrm{AA}^{\prime}}-J_{\mathrm{MM}^{\prime}} \\
\text { LAC2: }\left|\mathrm{ST} \mathrm{T}_{-}\right\rangle_{\mathrm{dtf}} \rightarrow\left|\mathrm{T}_{-} \mathrm{S}\right\rangle_{\mathrm{dtf}} \text { at }\left(\tilde{\nu}_{\mathrm{A}}-\tilde{\nu}_{\mathrm{M}}\right)=-J_{\mathrm{AA}^{\prime}}+J_{\mathrm{MM}^{\prime}} ; \\
\text { LAC3: }|\mathrm{SS}\rangle_{\mathrm{dtf}} \rightarrow\left|\mathrm{T}_{+} \mathrm{T}_{-}\right\rangle_{\mathrm{dtf}} \text { at }\left(\tilde{\nu}_{\mathrm{A}}-\tilde{\nu}_{\mathrm{M}}\right)=J_{\mathrm{AA}^{\prime}}+J_{\mathrm{MM}^{\prime}} \\
\text { LAC4: }|\mathrm{SS}\rangle_{\mathrm{dtf}} \rightarrow\left|\mathrm{T}_{-} \mathrm{T}_{+}\right\rangle_{\mathrm{dtf}} \text { at }\left(\tilde{\nu}_{\mathrm{A}}-\tilde{\nu}_{\mathrm{M}}\right)=-J_{\mathrm{AA}^{\prime}}-J_{\mathrm{MM}^{\prime} \cdot}
\end{gathered}
$$

To demonstrate how the LACs are formed we plotted the differences in energies, $\delta E$, for the levels given in eqn (13) as a function of the $\nu_{\mathrm{rf}}$ (assuming fixed $\nu_{1}$ ); at the LC point these differences reach their minima (given by the minimal splitting between the anti-crossing levels). In Fig. 3 such differences are shown for all four pairs of levels. The $\delta E\left(\nu_{\mathrm{rf}}\right)$ curves go through minima, which are grouped in two pairs, since the positions of LAC1 and LAC3 and also of LAC2 and LAC4 nearly coincide.

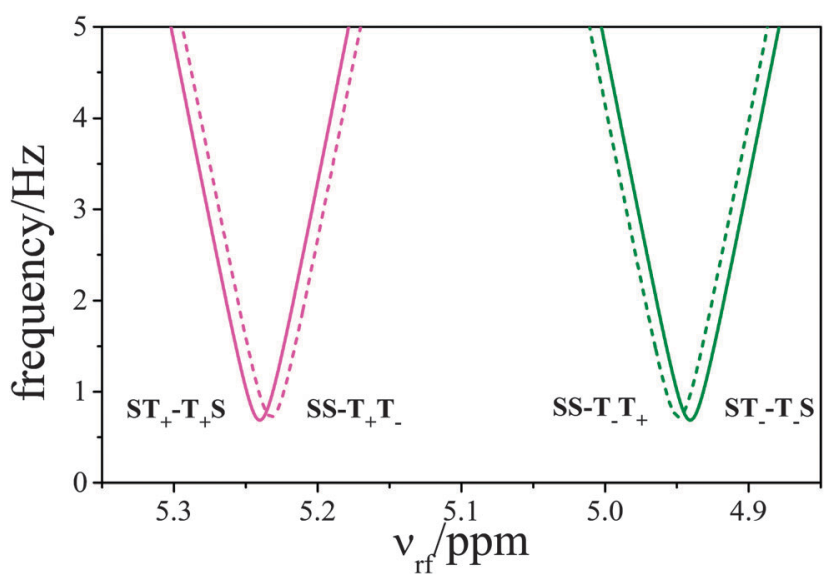

Fig. 3 Calculated energy differences between levels having LACs as a function of $\nu_{\mathrm{rf}}$. Here $\nu_{1}=3 \mathrm{kHz}$; the static magnetic field is $B_{0}=7 \mathrm{~T}$; energy is shown in $\mathrm{Hz}$; in the figure pairs of levels are assigned to specific LACs. Calculation is done for the $\mathrm{AA}^{\prime} \mathrm{MM}^{\prime}$-system with parameters taken from Table 1.
Let us find the frequency position of LACs for all four transitions. To do so, we rewrite $\nu_{\mathrm{rf}}$ as

$$
\nu_{\mathrm{rf}}=\frac{\nu_{\mathrm{A}}+\nu_{\mathrm{M}}}{2}+\nu_{\mathrm{off}}=\langle\nu\rangle+\nu_{\mathrm{off}}
$$

The new quantity, $\nu_{\text {off }}$, is thus an offset from the center of the spectrum. Typically $J$-couplings between protons do not exceed $20 \mathrm{~Hz}$, while the frequency difference, $\left(\nu_{\mathrm{A}}-\nu_{\mathrm{M}}\right)$, is of the order of a few $100 \mathrm{~Hz}$ (for instance, it is $600 \mathrm{~Hz}$ for two protons at 7 Tesla having a chemical shift difference of $2 \mathrm{ppm})$. Therefore it is safe to assume that $\nu_{\text {off }}$ is much smaller than $\left(\nu_{\mathrm{A}}-\nu_{\mathrm{M}}\right)$ and $\nu_{1}$, which allows one to write a simpler approximate expression for $\left(\tilde{\nu}_{\mathrm{A}}-\tilde{\nu}_{\mathrm{M}}\right)$

$$
\begin{aligned}
\tilde{\nu}_{\mathrm{A}}-\tilde{\nu}_{\mathrm{M}} & =\sqrt{\left(\Delta-\nu_{\mathrm{off}}\right)^{2}+\nu_{1}^{2}}-\sqrt{\left(\Delta+\nu_{\mathrm{off}}\right)^{2}+\nu_{1}^{2}} \\
& \approx-\frac{2 \Delta \nu_{\mathrm{off}}}{\sqrt{\Delta^{2}+\nu_{1}^{2}}}
\end{aligned}
$$

Here $\Delta=\left(\nu_{\mathrm{A}}-\nu_{\mathrm{M}}\right)$ is the difference in NMR frequencies of the two spin systems. The offsets, at which the LAC conditions are fulfilled, are then as follows:

$$
\begin{gathered}
\mathrm{LAC} 1 \text { and LAC3 }: \nu_{\mathrm{off}}=-\frac{\sqrt{\Delta^{2}+\nu_{1}^{2}}}{2 \Delta}\left(J_{\mathrm{AA}^{\prime}} \mp J_{\mathrm{MM}^{\prime}}\right) \\
\text { LAC2 and LAC4 }: \nu_{\mathrm{off}}=\frac{\sqrt{\Delta^{2}+\nu_{1}^{2}}}{2 \Delta}\left(J_{\mathrm{AA}^{\prime}} \pm J_{\mathrm{MM}^{\prime}}\right)
\end{gathered}
$$

To get to the LC point at small $\nu_{1}$ one has to step from the center of the spectrum, $\langle\nu\rangle$, by $\pm\left(J_{\mathrm{AA}^{\prime}} \pm J_{\mathrm{MM}^{\prime}}\right) / 2$ in frequency. As the $B_{1}$-field strength increases the LAC positions shift from the center of the spectrum (at $\nu_{1} \gg \Delta$ approximately as $\pm \nu_{1}\left(J_{\mathrm{AA}^{\prime}} \pm J_{\mathrm{MM}^{\prime}}\right) / 2 \Delta$ ) and the LAC regions become broader, see Fig. 4.

The theoretical analysis allows one to determine the LAC positions as a function of both, the frequency and the strength of the $B_{1}$-field. Now let us discuss what the consequences of mixing in the LAC regions are. Transitions occurring at LAC1

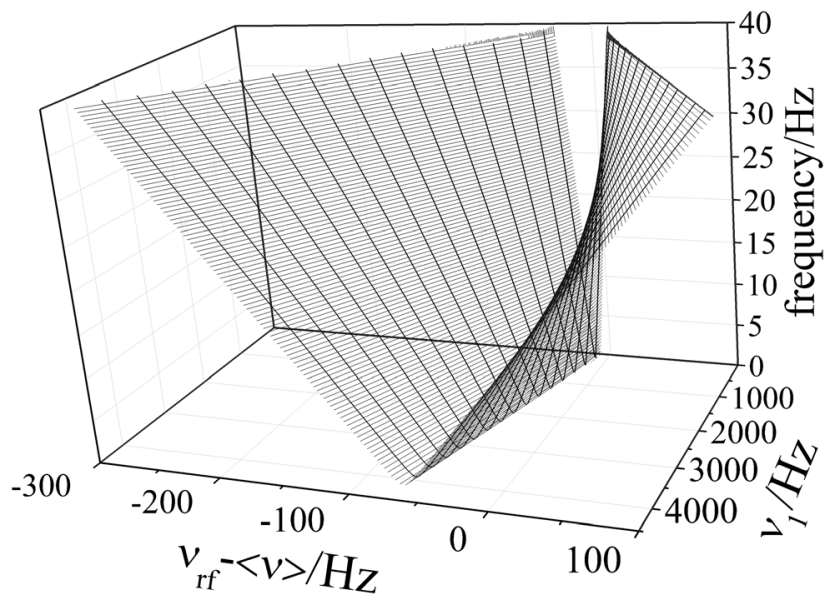

Fig. 4 Representation of the LAC between levels $S T_{+}$and $T_{+} S$ (LAC 1, see eqn (13)) of the $A A^{\prime} M M^{\prime}$-system in the DTF: dependence of the energy difference (measured in $\mathrm{Hz}$ ) between the anti-crossing levels on the off-set, $\left(\nu_{\mathrm{rf}}-\langle\nu\rangle\right)$, and the field strength $\nu_{1}$. Parameters of the spin system are taken from Table 1; the high static magnetic field is $B_{0}=7 \mathrm{~T}$. 
and LAC3 result in polarization of the $\mathrm{AA}^{\prime}$-spins along their effective field, $B_{\mathrm{A}}$, and polarization of the $\mathrm{MM}^{\prime}$-spins (of exactly the same size) opposite to the corresponding field vector, $B_{\mathbf{M}}$. The consequence of transitions at LAC2 and LAC4 is exactly the opposite. As shown below these simple rules allow one to understand the sign of the resulting polarization; the same method has been used to explain the magnetic field dependence of SABRE. ${ }^{34}$ However, in the present case we need to also take into account the time profile of switching the RF-field. In the general case one can do so only numerically; however, to get insight into the phenomenon under study we consider the resulting spin order in the two limiting situations of adiabatic and very fast (sudden) switching of the RF-field. As we will show, by choosing the switching time profile one can efficiently change the resulting spin order in a desired way. Thus, variation of the parameters of the $B_{1}$-field together with those of the switching is a powerful tool for manipulating PHIP.

\section{Effects of switching off the RF-field: non-adiabatic case}

Let us first discuss the non-adiabatic (sudden) switching case. In this situation the spin magnetization has no time to change during the switching. Thus, in order to calculate the resulting spin order it is sufficient to find the spin magnetizations in the DTF and then re-calculate them in the rotating non-tilted frame. In the DTF the spins acquire magnetizations, $\tilde{I}_{\mathrm{Az}}$ and $\tilde{I}_{\mathrm{Mz}}$, only along their effective fields, i.e., along the $B_{\mathrm{A}}$ and $B_{\mathrm{M}}$ axes. The resulting magnetizations in the non-tilted frame are then as follows:

$$
\begin{gathered}
I_{\mathrm{Az}}=\tilde{I}_{\mathrm{A} z} \sin \theta_{\mathrm{A}} ; \quad I_{\mathrm{A} x}=\tilde{I}_{\mathrm{A} z} \cos \theta_{\mathrm{A}} \\
I_{\mathrm{Mz}}=-\tilde{I}_{\mathrm{M} z} \sin \theta_{\mathrm{M}} ; \quad I_{\mathrm{M} x}=\tilde{I}_{\mathrm{M} z} \cos \theta_{\mathrm{M}} .
\end{gathered}
$$

The presence of the sine and cosine functions of $\theta_{\mathrm{A}}$ and $\theta_{\mathbf{M}}$ shows the effect of inclination of the effective fields. Hence, for calculating the resulting spin order in the non-adiabatic case the inclinations have to be taken into account. One can readily see that after rapidly switching off the RF-field not only longitudinal magnetization but also transverse magnetization is produced, which starts evolving and gives the Free Induction Decay (FID) signal without additional RF-pulses. For all the transitions occurring at the LC points one has exactly opposite $z$-magnetizations of the two spin groups in the DTF: $\tilde{I}_{\mathrm{M} z}=-\tilde{I}_{\mathrm{A} z}$. The polarization signs are as follows:

$$
\begin{aligned}
& \text { LAC1: } \left.|\mathrm{ST}\rangle_{+}\right\rangle_{\mathrm{dtf}} \rightarrow\left|\mathrm{T}_{+} \mathrm{S}\right\rangle_{\mathrm{dtf}} \tilde{I}_{\mathrm{A} z}>0, \tilde{I}_{\mathrm{Mz}}<0 ; \\
& \text { LAC2: } \left.|\mathrm{ST}\rangle_{-}\right\rangle_{\mathrm{dtf}} \rightarrow\left|\mathrm{T}_{-} \mathrm{S}\right\rangle_{\mathrm{dtf}} \tilde{I}_{\mathrm{Az}}<0, \tilde{I}_{\mathrm{M} z}>0 ; \\
& \text { LAC3: }|\mathrm{SS}\rangle_{\mathrm{dtf}} \rightarrow\left|\mathrm{T}_{+} \mathrm{T}_{-}\right\rangle_{\mathrm{dtf}} \tilde{I}_{\mathrm{A} z}>0, \tilde{I}_{\mathrm{M} z}<0 ; \\
& \text { LAC4: }|\mathrm{SS}\rangle_{\mathrm{dtf}} \rightarrow\left|\mathrm{T}_{-} \mathrm{T}_{+}\right\rangle_{\mathrm{dtf}} \tilde{I}_{\mathrm{A} z}<0, \tilde{I}_{\mathrm{M} z}>0 .
\end{aligned}
$$

These sign rules can be easily explained; let us do this, for instance, for LAC1. In the case of LAC1 initially the $\left|\mathrm{ST}_{+}\right\rangle_{\mathrm{dtf}}$ state is populated; after the transition to the $\left|\mathrm{T}_{+} \mathrm{S}\right\rangle_{\mathrm{dtf}}$ state the $\mathrm{AA}^{\prime}$-spins go to the state where they have positive projection on the $B_{\mathrm{A}}$ direction. The $\mathrm{MM}^{\prime}$-spins change their projection on the $B_{\mathrm{M}}$ direction from positive to zero. Thus, the variation of the spin projections, $\delta \tilde{I}_{\mathrm{A} z}$ and $\delta \tilde{I}_{\mathrm{M} z}$, in the DTF is positive and negative for the $\mathrm{AA}^{\prime}$ - and $\mathrm{MM}^{\prime}$-spins, respectively. Since initially both groups of spins have no net polarization (the $\mathrm{AA}^{\prime}$-spins are born in the singlet state and the $\mathbf{M M}^{\prime}$-spins have only negligible thermal polarization), after mixing at the LAC region the spin magnetizations, $\tilde{I}_{\mathrm{A} z}$ and $\tilde{I}_{\mathrm{Mz}}$, are equal to $\delta \tilde{I}_{\mathrm{A} z}$ and $\delta \tilde{I}_{\mathrm{Mz}}$. For this reason, hereafter we omit ' $\delta$ ' in the sign rules for magnetization.

However, the sign rule for the non-tilted frame can be different. For instance for LAC1 and LAC3 one gets:

$$
I_{\mathrm{A} z} \approx I_{\mathrm{M} z}>0, \quad I_{\mathrm{A} x} \approx-I_{\mathrm{M} x}<0
$$

For LAC2 and LAC4 the result is:

$$
I_{\mathrm{A} z} \approx I_{\mathrm{M} z}<0, \quad I_{\mathrm{A} x} \approx-I_{\mathrm{M} x}>0
$$

One can also generalize these sign rules for a situation where the RF-frequency is placed 'outside' the NMR spectrum, but the LAC conditions are also achieved. In this case for LAC1 and LAC3 one gets:

$$
I_{\mathrm{A} z} \approx-I_{\mathrm{M} z}>0, \quad I_{\mathrm{A} x} \approx-I_{\mathrm{M} x}<0
$$

and for LAC2 and LAC4

$$
I_{\mathrm{A} z} \approx-I_{\mathrm{M} z}>0, \quad I_{\mathrm{A} x} \approx-I_{\mathrm{M} x}>0
$$

These rules follow from the signs of the sine and cosine terms in eqn (17).

Hereafter we always assume that the RF-frequency is placed inside the NMR spectrum so that the LAC conditions are fulfilled at moderate RF-field strength. In this situation the $z$-magnetization components of spins in both groups in the non-tilted frame are always of the same sign. The $x$-magnetizations behave in the same way as the $z$-magnetizations in the DTF: they are the same in amplitude but different in sign. Thus, the inclination angles, $\theta_{\mathrm{A}}$ and $\theta_{\mathrm{M}}$, of the field axes in the DTF have to be taken into account when the behavior of spins in the vicinity of LACs is analyzed. To net-polarize the spins efficiently in the case of sudden switching it is necessary to keep the system in the LAC region.

Notably, when the RF-field strength is large we obtain $\cos \theta \rightarrow 1, \sin \theta \rightarrow 0$; consequently, transverse spin polarization is much larger than the longitudinal one: $\left|I_{\mathrm{A} x}, I_{\mathrm{M} x}\right| \gg\left|I_{\mathrm{A} z}, I_{\mathrm{M} z}\right|$. This situation is met in our experiments; hyperpolarizationenhanced FID signals are then obtained immediately after switching off the RF-field.

As shown below, in the case of adiabatic switching the conditions are different: the hyperpolarized spin system can be prepared outside the LAC region, but slow passage through the LAC region can nonetheless convert the initial singlet order of the A-spins into observable polarization.

\section{Effects of switching off the RF-field: adiabatic case}

Now let us consider the adiabatic case. In this situation the state populations follow the eigen-states, which gradually change with time. To find the final state populations it is thus necessary to correlate the spin states of the system. Here, by 'correlation of states' we mean that for a manifold of coupled spin states the population of the level with highest energy under spin locking conditions coincides with that of the level 
with highest energy in the absence of the RF-field; the population of the second highest level at strong RF-field goes to that of the second highest after the RF-field has been switched off, and so on. Methods based on adiabatic passage are frequently used in $\mathrm{NMR}^{47,49-51}$ (in many cases, adiabatic passage through the Hartmann-Hahn condition is exploited, see ref. 48 for more details) and in optical spectroscopy. ${ }^{52}$

Interestingly, when the sign of the $z$-components of the effective fields $B_{\mathrm{A}}$ and $B_{\mathrm{M}}$ is opposite (which is the case when the RF-frequency is placed between the frequencies of the A-spins and M-spins) upon lowering $B_{1}$ the field vectors become parallel and anti-parallel to the $z$-axis. Then the anti-parallel $z$-projections of the spins in the DTF result in parallel $z$-projections in the non-tilted frame: both spins can be net-polarized with the same polarization sign (either absorptive or emissive depending on the particular LAC chosen). Let us show how it works for different LAC points. For instance, the transition $\left|\mathrm{ST}_{+}\right\rangle_{\mathrm{dtf}} \rightarrow$ $\left|\mathrm{T}_{+} \mathrm{S}\right\rangle_{\mathrm{dtf}}$ occurring at LAC1, see eqn (13), corresponds to the transition $\left|S T_{-}\right\rangle \rightarrow\left|\mathrm{T}_{+} \mathrm{S}\right\rangle$ in the non-tilted frame. As a consequence, both spins acquire positive $z$-polarization: $I_{\mathrm{A} z} \approx I_{\mathrm{M} z}>0$. Likewise, the other LAC-induced transitions result in the following transitions in the non-tilted frame:

$$
\begin{aligned}
& \text { LAC2: }\left|\mathrm{ST}_{+}\right\rangle \rightarrow\left|\mathrm{T}_{-} \mathrm{S}\right\rangle, \quad I_{\mathrm{A} z}=I_{\mathrm{M} z}<0 ; \\
& \text { LAC3: }|\mathrm{SS}\rangle \rightarrow\left|\mathrm{T}_{+} \mathrm{T}_{+}\right\rangle, \quad I_{\mathrm{A} z}=I_{\mathrm{M} z}>0 ; \\
& \text { LAC4: }|\mathrm{SS}\rangle \rightarrow\left|\mathrm{T}_{-} \mathrm{T}_{-}\right\rangle, \quad I_{\mathrm{A} z}=I_{\mathrm{M} z}<0 ;
\end{aligned}
$$

Thus, it becomes possible to net-polarize the spins along the $z$-axis with the same sign of polarization. This is a generalization of the method discussed in our earlier work, ${ }^{42}$ where a two-spin system was net-polarized in an analogous way by using adiabatic switching of the RF-field with properly set frequency. The physical origin of this effect is also similar to that of the one discussed here. In our present case the difference is that the spin system is not only prepared at a very strong RF-field but that the RF-field parameters, $\nu_{\mathrm{rf}}$ and $\nu_{1}$, are carefully adjusted to guarantee the LAC conditions. This gives an additional advantage, namely that the resulting polarization pattern can be manipulated by both, spin mixing at the LAC region and correlating spin states in the presence and in the absence of the RF-field. In contrast to the non-adiabatic case, there is no transverse magnetization in the system but only longitudinal one. Thus, to obtain NMR spectra with enhanced lines it is necessary to apply an RF-pulse to observe the FID.

It is important to note that one can net-polarize the spins not only in the case of preparation directly at an LC point but also by preparation away from an LAC with subsequent adiabatic passage through the LAC. Let us assume, for instance that the system is initially prepared in the $|\mathrm{SS}\rangle_{\mathrm{dtf}}$-state and passes through an LAC with the $\left|\mathrm{T}_{+} \mathrm{T}_{-}\right\rangle_{\mathrm{dtf}}$-state in the DTF. When the adiabatic conditions are fulfilled after the passage the system goes to the new state, $\left|\mathrm{T}_{+} \mathrm{T}_{-}\right\rangle_{\mathrm{dtf}}$, since the crossing is avoided and the system always stays in the energetically uppermost or lowermost state. Consequently, the $\left|\mathrm{T}_{+} \mathrm{T}_{-}\right\rangle_{\mathrm{dtf}}$-state acquires the population, which is identical to the initial population of the

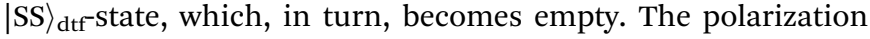
transfer is even more efficient as compared to the case of preparation at the LC point. When the states are mixed at the LAC region the population is shared evenly among then, while in the adiabatic passage case $100 \%$ of the population is transferred to the new state. Thus, to transfer the singlet spin order of the $\mathrm{AA}^{\prime}$-spins it is sufficient and even preferable to pass adiabatically through the LAC. Adiabatic field switching is also preferable because the adjustment of $\nu_{\mathrm{rf}}$ and $\nu_{1}$ for fulfilling the LAC conditions is rather robust: instead of going to the LAC region (which is a prerequisite for non-adiabatic switching) it is sufficient only to go through the LAC region during the switching. Such an advantage of adiabatic passage through an LAC region can also be used for spin order conversion in other experiments. ${ }^{53}$

In this section we do not illustrate the general results by theoretical calculations; instead in Section IV we describe in detail the experimental data and model them. As shown below, experiments completely confirm our expectations: we demonstrate the effects of LACs and show that mixing at the LAC regions provides efficient polarization of the spin system. The LAC conditions, polarization patterns and polarization sign are in full accordance with our theoretical predictions. We also study in detail the effects of switching off the RF-field and demonstrate the possibility of manipulating the spectral pattern by choosing the switching time profile. We perform the experiments for molecules having the same symmetry as the one described here. An additional property of our experimental system is that one of the two couplings, $J_{\mathrm{AA}^{\prime}}$ and $J_{\mathrm{MM}^{\prime}}$, is much larger than the other one: $J_{\mathrm{AA}^{\prime}} \gg J_{\mathrm{MM}^{\prime}}$. As a consequence, the LC points are grouped in two pairs as the LAC conditions become identical for them:

$$
\begin{aligned}
& \text { LAC1,3: }\left|\mathrm{ST}_{+}\right\rangle_{\mathrm{dtf}} \rightarrow\left|\mathrm{T}_{+} \mathrm{S}\right\rangle_{\mathrm{dtf}} \text { and }|\mathrm{SS}\rangle_{\mathrm{dtf}} \rightarrow\left|\mathrm{T}_{+} \mathrm{T}_{-}\right\rangle_{\mathrm{dtf}} \\
& \text { at }\left(\tilde{\nu}_{\mathrm{A}}-\tilde{\nu}_{\mathrm{M}}\right) \approx J_{\mathrm{AA}^{\prime}} \\
& \text { LAC2,4: }\left|\mathrm{ST} \mathrm{T}_{-}\right\rangle_{\mathrm{dtf}} \rightarrow\left|\mathrm{T}_{-} \mathrm{S}\right\rangle_{\mathrm{dtf}} \text { and }|\mathrm{SS}\rangle_{\mathrm{dtf}} \rightarrow\left|\mathrm{T}_{-} \mathrm{T}_{+}\right\rangle_{\mathrm{dtf}} \\
& \text { at }\left(\tilde{\nu}_{\mathrm{A}}-\tilde{\nu}_{\mathrm{M}}\right) \approx-J_{\mathrm{AA}^{\prime}} \text {; }
\end{aligned}
$$

This makes the treatment simpler; it is also important to emphasize that spin mixing at LAC1 and LAC3 results in the same sign and magnitude of spin polarization; the same holds for the LAC2 and LAC4. Thus, effectively the spin system is a system with two different LACs.

\section{Materials and methods}

\section{Experimental protocol}

Experiments were performed according to the scheme shown in Fig. 5. First, the sample was bubbled by dihydrogen gas enriched in its para spin isomer directly in the NMR sample tube for a time period $\tau_{\mathrm{b}}=2 \mathrm{~s}$. After that during a time period of less than $0.5 \mathrm{~s}$ the gas flow was stopped so that chemical reactions, which lead to PHIP formation, run for a time period $\tau_{\mathrm{r}}=7.5 \mathrm{~s}$. After that an RF-pulse with the amplitude $B_{1}$ and the carrier frequency $\nu_{\mathrm{rf}}$ was applied for a time period $\nu_{\mathrm{rf}}=1 \mathrm{~s}$. 

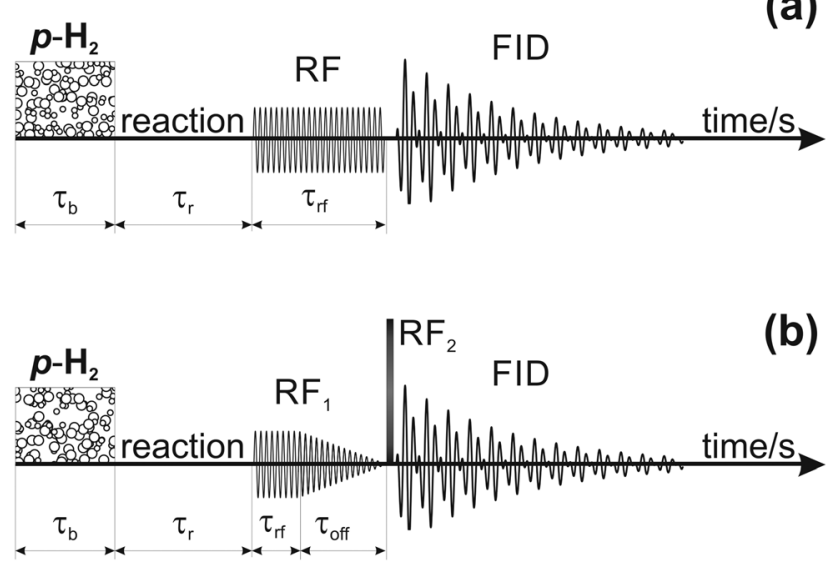

Fig. 5 Timing scheme of the experiment in the case of non-adiabatic (a) and adiabatic (b) fading of the RF-field. Here para- $\mathrm{H}_{2}$ gas is bubbled through the sample for a time period $\tau_{\mathrm{b}}$, then the hydrogenation reaction is run for a time period $\tau_{r}$. After that the spin-locking field is switched on. In case (a) the spin-locking field, RF, is on during the time period $\tau_{\mathrm{rf}}$; then it is switched off instantaneously and the FID signal is detected without additional excitation pulses. In case (b) the spin-locking field, $\mathrm{RF}_{1}$, is on during the time period $\tau_{\mathrm{rf}}$, then it is switched off adiabatically during the time $\tau_{\text {off }}$ and an RF-pulse $\left(\mathrm{RF}_{2}\right)$ is applied to detect the FID signal.

In the case of non-adiabatic fading of the RF-field (the RF pulse was switched off faster than $40 \mu \mathrm{s}$ ) the FID was measured without any additional detecting RF-pulse. In the adiabatic case the RF-field was switched off linearly during a time period $\tau_{\text {off, }}$ which was varied from $40 \mu \mathrm{s}$ to $5 \mathrm{~s}$ (for fully adiabatic field switching). To observe the NMR signals a $\pi / 2$ RF-pulse was applied and the FID was detected.

Experiments were carried out using a $300 \mathrm{MHz}$ NMR spectrometer. The in situ bubbling system was the same as the one described earlier. ${ }^{41}$

\section{Chemical compounds}

Dimethyl acetylenedicarboxylate and acetone- $\mathrm{d}_{6}$ were purchased from Sigma-Aldrich. Catalyst [1,4-bis(diphenylphosphino)butane](1,5-cyclooctadiene)rhodium(I) tetrafluoroborate was purchased from ABCR (Germany). In all experiments we used the mixture of dimethyl acetylenedicarboxylate $(0.26 \mathrm{M})$ and catalyst $(2.2 \mathrm{mM})$ in acetone- $\mathrm{d}_{6}$. The mixture was used without additional purification. The scheme of the hydrogenation reaction, which leads to PHIP formation, is given in Chart 1.

\section{Calculation scheme}

We performed our calculation for 4 protons even though maleic acid dimethyl ester has 8 coupled protons. As shown before ${ }^{35,37}$ the protons of the $\mathrm{CH}_{3}$ group here can be effectively replaced by a single proton. The following initial density matrix was taken

$$
\rho(-0)=\left(\frac{E}{4}-\left(\hat{\mathrm{I}}_{\mathrm{A}} \cdot \hat{\mathrm{I}}_{\mathrm{A}^{\prime}}\right)\right) / 4
$$

This density matrix was projected on the eigen-basis of the high-field spin Hamiltonian in the absence of the RF-field. As a consequence, the eigen-states are populated with respect to the
Table 1 NMR parameters (J-couplings and chemical shifts) of the $\mathrm{AA}^{\prime} \mathrm{MM}^{\prime}$-system modeling maleic acid dimethyl ester

\begin{tabular}{lllll}
\hline$J / \mathrm{Hz}$ & $\mathrm{A}$ & $\mathrm{A}^{\prime}$ & $\mathrm{M}$ & $\mathrm{M}^{\prime}$ \\
\hline $\mathrm{A}$ & & - & - & - \\
$\mathrm{A}^{\prime}$ & 12 & & - & - \\
$\mathrm{M}$ & 1 & 0.3 & & - \\
$\mathrm{M}^{\prime}$ & 0.3 & 1 & 0 & \\
$\delta / \mathrm{ppm}$ & 6.416 & 6.416 & 3.765 & 3.765 \\
\hline
\end{tabular}

singlet character of the $\mathrm{AA}^{\prime}$-spins; all spin coherences between the states were neglected. This is because hydrogenation reactions typically proceed for an extended time interval so that all coherences are washed out. ${ }^{31,43}$ The resulting density matrix (when relaxation effects are neglected) then represents the density matrix at $t=\tau_{\mathrm{b}}+\tau_{\mathrm{r}}$, that is $\rho\left(\tau_{\mathrm{b}}+\tau_{\mathrm{r}}\right)$.

After that an RF-pulse characterized by the $\nu_{1}$ and $\nu_{\mathrm{rf}}$ values of choice is applied. In the presence of the RF-field the Hamiltonian and, consequently, the eigen-basis change again. To take this into account we again projected the density matrix $\rho\left(\tau_{\mathbf{b}}+\tau_{\mathrm{r}}\right)$ on the new basis and removed all off-diagonal elements (coherences), since the hydrogenation continues. The resulting density matrix is then $\rho\left(\tau_{\mathrm{b}}+\tau_{\mathrm{r}}+\tau_{\mathrm{rf}}\right)$.

In the case of non-adiabatic switching this matrix has to be used to evaluate the expectation values of all spin magnetization components: $\left\langle I_{\mathrm{A} x, z}\right\rangle=\operatorname{Tr}\left\{\hat{1}_{\mathrm{A} x, z} \rho\left(\tau_{\mathrm{b}}+\tau_{\mathrm{r}}+\tau_{\mathrm{rf}}\right)\right\}$ and $\left\langle I_{\mathrm{M} x, z}\right\rangle=$ $\operatorname{Tr}\left\{\hat{I}_{\mathrm{M} x, z} \rho\left(\tau_{\mathrm{b}}+\tau_{\mathrm{r}}+\tau_{\mathrm{rf}}\right)\right\}$. The values of $\left\langle I_{\mathrm{A} x}\right\rangle$ and $\left\langle I_{\mathrm{M} x}\right\rangle$ correspond to the intensities in the spectra measured without additional RF-pulses.

In the case of adiabatic field switching an additional step is required because the density matrix of the spin system changes during the switching. To take this effect into account we divided the time interval from $t=\tau_{\mathrm{b}}+\tau_{\mathrm{r}}+\tau_{\mathrm{rf}}$ to $t=\tau_{\mathrm{b}}+\tau_{\mathrm{r}}+$ $\tau_{\text {rf }}+\tau_{\text {off }}$ in $N$ small steps of duration $\delta t=\tau_{\text {off }} / N$. The evolution is described by the following operator

$$
\hat{S}=\prod_{n=1}^{N} \exp \left(-2 \pi i \hat{H}_{\mathrm{rf}}\left(\nu_{1}\left(t_{n}\right), v_{\mathrm{rf}}\right) \delta t\right)
$$

and the density matrix after the switching is

$$
\rho\left(\tau_{\mathrm{b}}+\tau_{\mathrm{r}}+\tau_{\mathrm{rf}}+\tau_{\text {off }}\right)=\hat{S} \rho\left(\tau_{\mathrm{b}}+\tau_{\mathrm{r}}+\tau_{\mathrm{rf}}\right) \hat{S}^{-1}
$$

Here $\hat{H}_{\mathrm{rf}}\left(\nu_{1}\left(t_{n}\right), \nu_{\mathrm{rf}}\right)$ is the Hamiltonian (1) at the $n$-th instant of time, $t_{n}=n \delta t$ so that the RF-field strength is equal to $\nu_{1}\left(t_{n}\right)$. Typically, $\delta t=5 \mathrm{~ms}$ was taken; we made sure that the result of our calculation was independent of $\delta t$ in the limit of large $N$.

The parameters of the spin system used in our calculation (chemical shifts and spin-spin couplings) are shown in Table 1. When comparison between theory and experiment is made the experimental dependences of polarization on $\nu_{1}$ and $\nu_{\mathrm{rf}}$ are always normalized to the calculated curves.

\section{Results and discussion}

As mentioned above we performed experiments by systematically varying the parameters of the RF-field, i.e., $\nu_{1}$ and $\nu_{\mathrm{rf}}$, to match the LAC conditions. Studying the dependence on the 
RF-field parameters is necessary to understand under what conditions the spin system can be brought to LAC regions and how to make better use of spin mixing in the LAC regions. In accordance with previous studies ${ }^{35-37}$ we have found that in the case under study the initial spin order can be transformed into NMR observables only at the LAC regions. We also varied the switching times to demonstrate effects of adiabatic $v s$. non-adiabatic variation of the Hamiltonian. This consideration is of importance for efficiently converting the initial spin order into spin magnetization.

Let us first discuss the simpler case of non-adiabatic switching.

\section{Case of non-adiabatic fading of the RF-field}

To fulfill the conditions of non-adiabatic switching we switched off the RF-field in less than $40 \mu$ s before accumulation of the FID was started. As mentioned, immediately after the switch there is transverse magnetization in the system, thus the FID can be detected without applying additional RF-pulses. This is a clear indication of non-adiabatic switching.

Fig. 6 shows the spectra obtained by the Fourier transformation of the FID signals originating from this transverse polarization. The two spectra shown in this figure have been obtained for two different values of $\nu_{\mathrm{rf}}$ (to match the level crossing conditions) and for sufficiently strong $\nu_{1}$ (to provide the 'coupling' condition, i.e., efficient mixing of the crossing states). As expected from the theoretical analysis the proper $\nu_{\mathrm{rf}}$ values are close to the center of the NMR spectrum, $\langle\nu\rangle$, but slightly detuned from it, see eqn (16). To fulfill the LAC condition in the DTF the frequency difference is set equal to $\pm J_{\mathrm{AA}^{\prime}}$. When the choice of $\nu_{\mathrm{rf}}$ at a given $\nu_{1}$ is improper, the PHIP spectrum vanishes. In both cases the $\mathrm{AA}^{\prime}$-spins and $\mathrm{MM}^{\prime}$-spins exhibit polarization of opposite sign, fully consistent with the theory. The spectral patterns are thus of the E/A (low-field emission and high-field absorption) or A/E (low-field absorption and high-field emission) type. By changing the sign of the offset, $\nu_{\text {off }}$, one can reverse the sign of polarization, which is

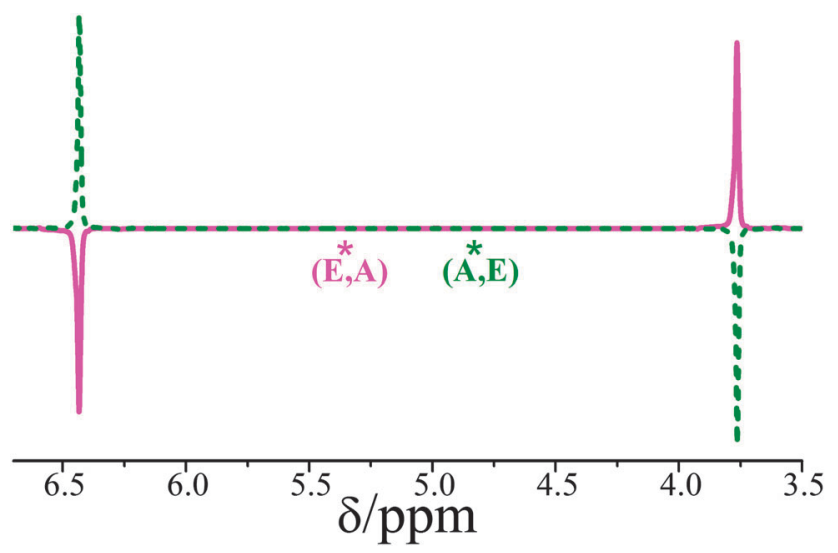

Fig. 6 PHIP spectra of maleic acid dimethyl ester obtained at $B_{0}=7 \mathrm{~T}$ after non-adiabatic reduction of the RF-field. The two spectra have been obtained for $\nu_{\mathrm{rf}}=5.36 \mathrm{ppm}$ (solid line) and $4.83 \mathrm{ppm}$ (dashed line). The $\nu_{\mathrm{rf}}$ positions are denoted by asterisks as well as the corresponding spectral patterns, which are emission/absorption $(E, A)$ and absorption/emission $(A, E)$. The $B_{1}$-field amplitude was $5.3 \mathrm{kHz}$; no additional RF-pulses have been applied to obtain the spectra.

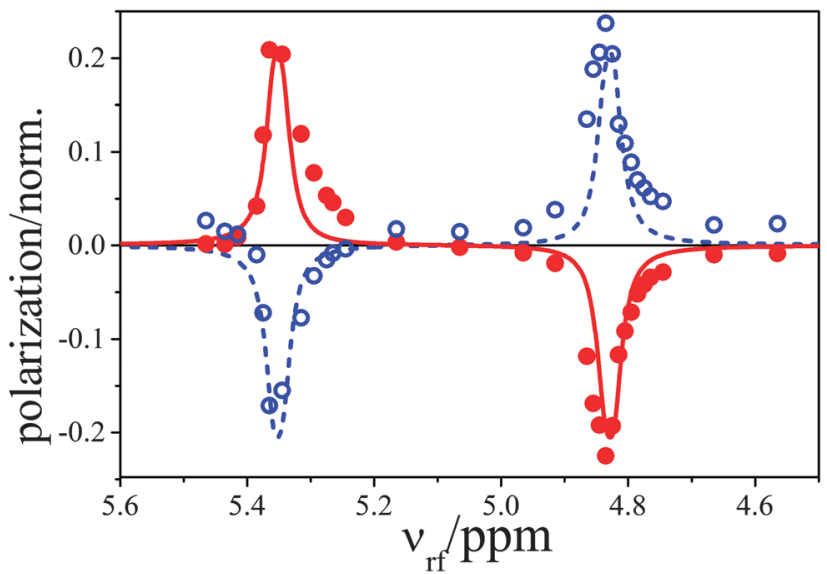

Fig. 7 Dependence of PHIP (integrated NMR line intensity) of the $A A^{\prime}$ protons (vinyl, full circles) and $\mathrm{MM}^{\prime}$-protons (methyl, open circles) on the frequency $\nu_{\mathrm{rf}}$. Here, the RF-amplitude $\nu_{1}$ is $5.3 \mathrm{kHz}$, the high static magnetic field is $B_{0}=7 \mathrm{~T}$; the RF-field is switched off rapidly; no additional RF-pulses are applied to obtain the spectra. Solid and dashed lines show the theoretical results.

also in accordance with the theoretical treatment. This is because the resulting polarization sign depends on the particular LAC that was involved in the spin mixing process.

We also studied in detail the frequency dependence of the PHIP magnetization, which is shown in Fig. 7, where the integrated intensity of the spectral lines is plotted as a function of $\nu_{\mathrm{rf}}$. It is readily seen that observable spin order is formed only when the states are mixed in the LAC region: the dependence of PHIP on $\nu_{\mathrm{rf}}$ exhibits two sharp features, which are centered at the specific frequencies, which correspond to the LACs in the DTF. The smaller $\nu_{1}$ is, the narrower these features are; also the distance between the $\nu_{\mathrm{rf}}$ values, which give the peaks in Fig. 7, is smaller (because the LACs are less separated with respect to $\nu_{\mathrm{rf}}$, see Fig. 4). The experimental results are in very good agreement with the theoretical treatment: the positions of the features and their widths are perfectly reproduced by the calculation.

It is also of interest to study the dependence on $\nu_{1}$ at a fixed $\nu_{\mathrm{rf}}$ value. Such dependence is shown in Fig. 8. When the RF-field is very low there is no polarization observed even when the levels cross in the DTF. In this situation the mixing in the DTF becomes very inefficient; at small $\nu_{1}$ we have $\cos ^{2} \Theta \rightarrow 0$ in eqn (11) and there is no transfer of singlet spin order into observable magnetization. In the example shown in Fig. 8 at $\nu_{1}=0$ the polarization is zero, then, as the field increases, the system passes through a LAC and spin polarization emerges. However, at very large $\nu_{1}$ values the polarization vanishes also because the spin system is driven away from the LAC region: the difference in frequency, $\tilde{\nu}_{\mathrm{A}}$ $-\tilde{\nu}_{\mathrm{M}}$, in the DTF becomes very small and cannot match $\pm J_{\mathrm{AA}^{\prime}}$. As a consequence, as a function of $\nu_{1}$ the polarization goes through a positive or negative maximum. Again, the experimental results are in full accordance with the theory.

Thus, qualitatively similar results can be obtained as compared to the case of varying the static magnetic field: at LAC regions one can net-polarize the $\mathrm{AA}^{\prime}$-spins and $\mathrm{MM}^{\prime}$-spins; the sign of polarization is opposite for both groups (see ref. 35 and Fig. 1). 


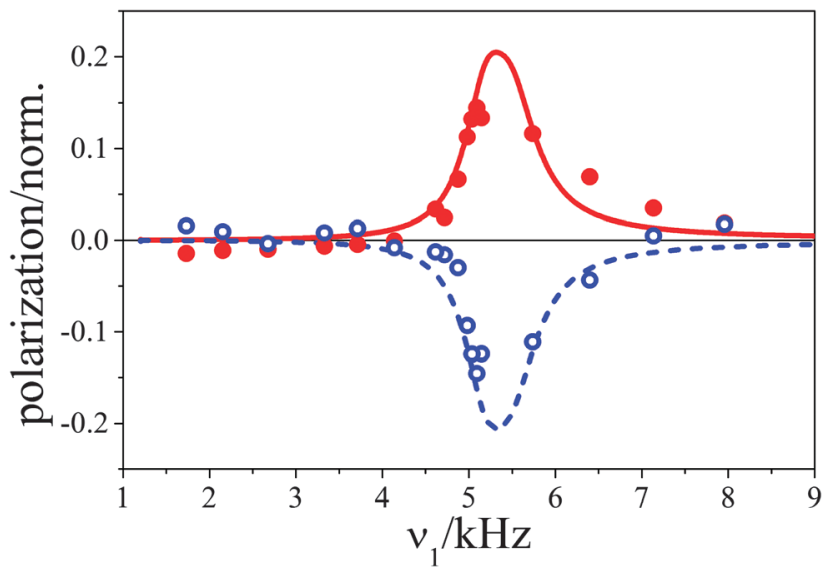

Fig. 8 Dependence of PHIP of the $A A^{\prime}$-protons (vinyl, full circles) and $M M^{\prime}$-protons (methyl, open circles) on the RF-field amplitude $\nu_{1}$. Here the value of $\nu_{\mathrm{rf}}$ was $5.35 \mathrm{ppm}$, the high static magnetic field is $B_{0}=7 \mathrm{~T}$; the RF-field was switched off non-adiabatically; no additional RF-pulses have been applied to obtain the spectra. Solid and dashed lines show the theoretical results.

The difference is that (i) one can change the polarization phase by varying $\nu_{\mathrm{rf}}$ and (ii) polarization is detected without additional RF-pulses. The latter factor is a fingerprint of non-adiabatic switching of the RF-field.

Now let us turn to the case of adiabatic switching.

\section{Case of adiabatic fading of the RF-field}

To fulfill the conditions of adiabatic switching we systematically varied the switching time; when the switching time, $\tau_{\text {off }}$, was chosen so long that the resulting polarization showed almost no change at variation of $\tau_{\text {off }}$ we assumed that the adiabatic limit has been reached. We also checked the adiabaticity by numerical calculations: the experimental values for the obtained $\tau_{\text {off }}$ were consistent with the calculated ones. In contrast to the non-adiabatic case the spectra can only be detected by applying an RF-pulse, since the spin order after the RF-field reduction is purely longitudinal.

Fig. 9 presents the PHIP spectra obtained for two different frequencies, $\nu_{\mathrm{rf}}$, after adiabatic fading of the RF-field. As previously (see Fig. 6) we have chosen $\nu_{\mathrm{rf}}$ such that the LAC conditions are fulfilled. As predicted by the theory in both cases spins have the same polarization sign; this is also a fingerprint of adiabatic switching. The sign of polarization can be reverted by going to a different LAC: for the two cases shown in Fig. 9 all NMR lines are either positive or negative.

The dependence of the PHIP magnetization on the pumping frequency $\nu_{\text {rf }}$ shown in Fig. 10 also has two sharp features, which correspond to the LACs in the DTF. The two features have opposite signs as for the two LACs different spin states are mixed. However, in contrast to the non-adiabatic case, the sign of polarization is the same for all protons. The experimental results are in very good agreement with the theory. It is important to note that the width of the peaks in Fig. 10 strongly depends on $\nu_{1}$ : the peaks become broader with increasing RF-field strength. This shows that when $\nu_{1}$ is large it is possible

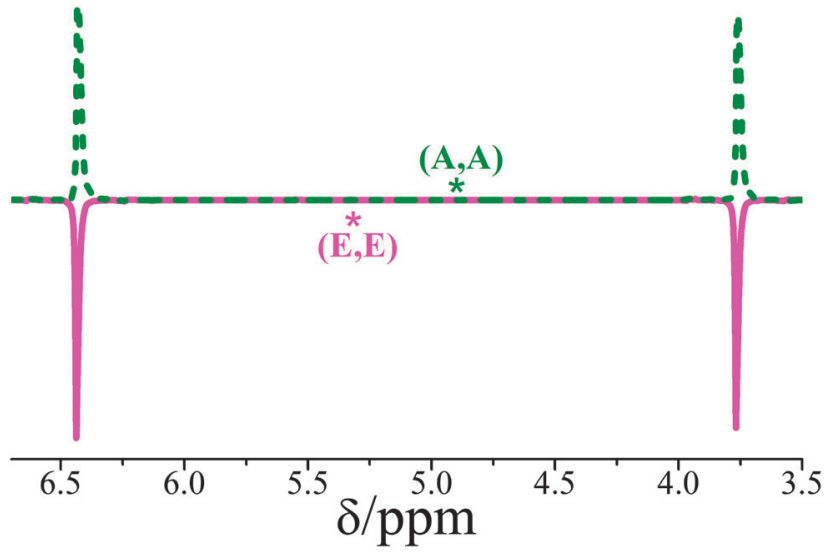

Fig. 9 PHIP spectra of maleic acid dimethyl ester obtained after adiabatic reduction of the RF-field. The two spectra have been obtained for $\nu_{\mathrm{rf}}=$ $5.32 \mathrm{ppm}$ (solid line) and $4.85 \mathrm{ppm}$ (dashed line). The $\nu_{\mathrm{rf}}$ positions are denoted by asterisks as well as the corresponding spectral patterns, which are emission/emission (E,E) and absorption/absorption (A,A). The $B_{1}$-field amplitude was $4.9 \mathrm{kHz}$, the high static magnetic field was $B_{0}=7 \mathrm{~T} ; \mathrm{a} \pi / 2$ RF-pulse was used to obtain the spectra.

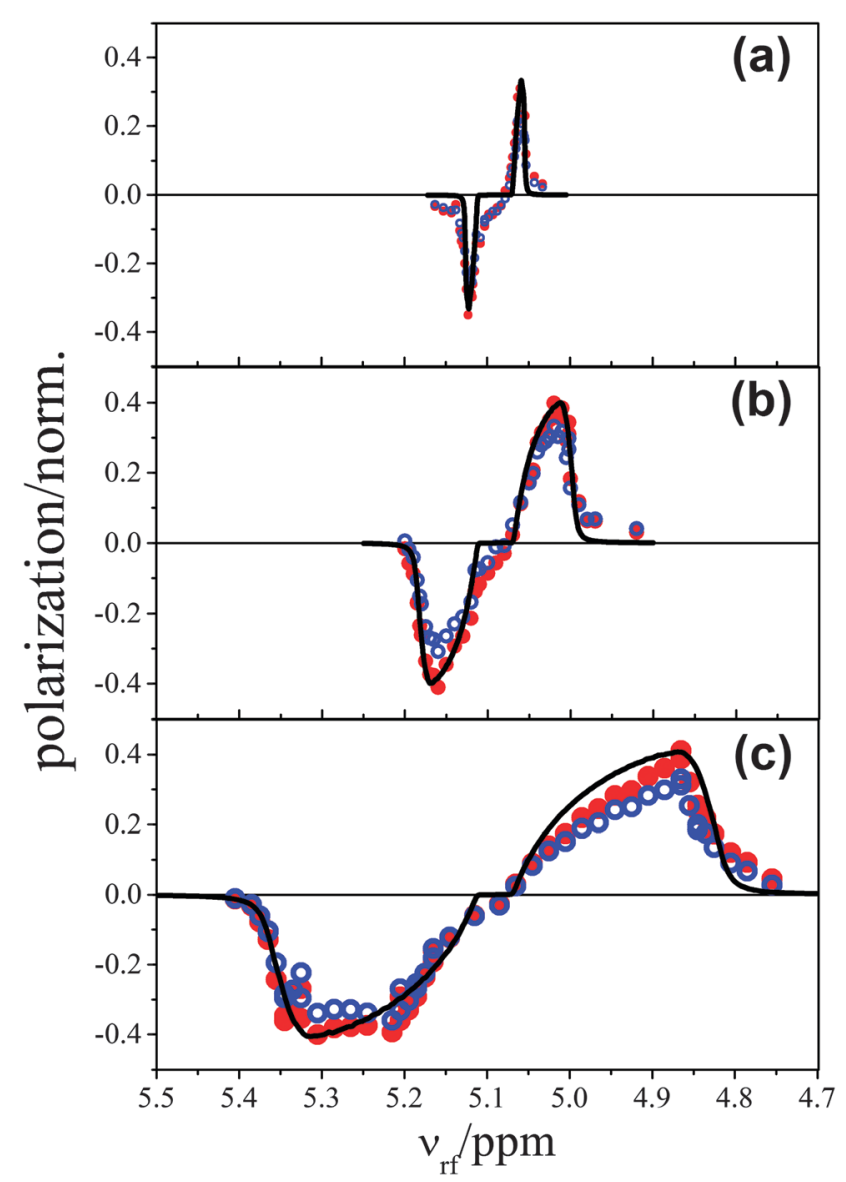

Fig. 10 Dependence of PHIP of the $A A^{\prime}$-protons (vinyl, full circles) and $M M^{\prime}$ - protons (methyl, open circles) on frequency $\nu_{\mathrm{rf}}$. Here the value of $\nu_{1}$ was $590 \mathrm{~Hz}(\mathrm{a}), 1.8 \mathrm{kHz}$ (b) and $5.4 \mathrm{kHz}$ (c); the high static magnetic field was $B_{0}=7 \mathrm{~T}$; the RF-field was switched off adiabatically during the time $\tau_{\text {off }}=5 \mathrm{~s}$; a $\pi / 2$ RF-pulse was used to obtain the spectra. Solid line shows the theoretical result. 


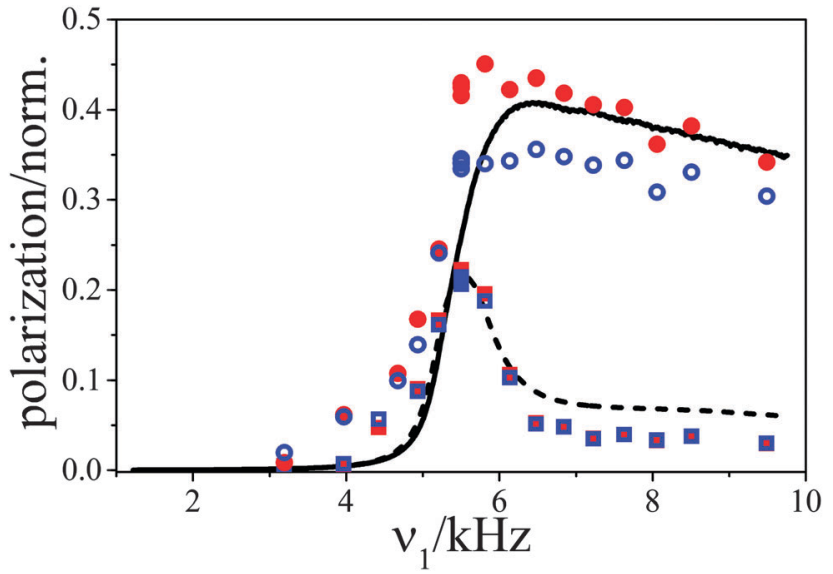

Fig. 11 Dependence of PHIP of the $A A^{\prime}$-protons (vinyl, full symbols) and $M M^{\prime}$-protons (methyl, open symbols) on the RF-field amplitude $\nu_{1}$. Here the value of $\nu_{\mathrm{rf}}$ was $4.83 \mathrm{ppm}$; a $\pi / 2 \mathrm{RF}$-pulse was used for detection; the high static magnetic field was $B_{0}=7 \mathrm{~T}$. The fading time $\tau_{\text {off }}$ of the RF-field was varied: $\tau_{\text {off }}=5 \mathrm{~s}$ (circles) and $\tau_{\text {off }}=0.5 \mathrm{~s}$ (squares). Lines show the theoretical results (solid lines for $\tau_{\text {off }}=5 \mathrm{~s}$ and dashed lines for $\tau_{\text {off }}=5 \mathrm{~s}$ ).

to transfer polarization even when initially, at $t=\tau_{\mathrm{b}}+\tau_{\mathrm{r}}+\tau_{\mathrm{rf}}$, the system is out of the LAC regions. In this situation the polarization is transferred because during switching off the RF-field the system passes through the LACs in an adiabatic fashion. As a consequence, the populations of the crossing levels are mixed and spin order is transferred. In such a situation the choice of $\nu_{\mathrm{rf}}$ can be done much less carefully than in the non-adiabatic case (where one should go exactly to a LAC region); hence such a method of polarization transfer is more simple and robust. Again, this result is in full agreement with our theoretical treatment.

Finally we investigated the dependence on the RF-amplitude $\nu_{1}$ at a fixed $\nu_{\mathrm{rf}}$ value. The results are shown in Fig. 11. As in the case of sudden switching, no spin polarization is formed at low RF-field. Upon increasing the RF-field spin polarization appears, but it has a different dependence on $\nu_{1}$ as compared to the case of sudden switching. Notably, there is hardly any decrease of polarization at the high end of $\nu_{1}$. This is a consequence of adiabatic switching. Although at large $\nu_{1}$ the system is away from the LAC point it passes through the LAC upon reduction of the field. When the reduction is adiabatic the passage through the LAC point net-polarizes the spins. Thus, in the limiting case of fully adiabatic switching the dependence is expected to reach a plateau at large $\nu_{1}$. Such a dependence is indeed observed for sufficiently long $\tau_{\text {off }}$. When $\tau_{\text {off }}$ is reduced, the height of the plateau becomes smaller and a maximum appears at an intermediate $\nu_{1}$ value. This behavior indicates that the switching is no longer purely adiabatic: the effect is clearly seen from the $\nu_{1}$ dependence (although the spectral patterns, i.e., two positive lines, remain unchanged in this case). In the limiting case of very fast switching the polarization at large $\nu_{1}$ goes to zero. Also these experimental results are well described by the theory.

\section{Conclusions}

We have demonstrated efficient polarization transfer in NMR experiments where a coupled spin system is brought to an LC point in the rotating frame by switching on an RF-field with properly set amplitude and frequency. Our study confirms previous results on spin order transfer under spin-locking conditions $^{37,42}$ and generalizes them: in addition to mixing of spin states at LAC points we have studied how the time profile of passing through an LAC affects the observed polarization, thus providing a tool to further manipulate it.

We have generalized the theoretical description of the problem establishing the conditions for having an LAC in the rotating frame, showing that conditions of (i) matching (coincidence of energy levels of the main Hamiltonian of the system) and (ii) coupling (presence of a perturbation which turns a level crossing into an avoided crossing) have to be met. In addition, an important factor is the inclination of the effective field vector in the rotating frame, which has to be taken into account for interpreting the experimental data. Since the observed polarization is associated with well-defined LACs one can formulate simple qualitative sign rules for the observed polarization in very much the same way as it was done ${ }^{34}$ for the case of SABRE. The experimental data obtained for PHIP transfer in symmetric molecules $\left(\mathrm{AA}^{\prime} \mathrm{MM}^{\prime}\right)$ are in perfect agreement with the theoretical treatment. In particular, we studied in detail how the LAC conditions depend on the experimentally accessible parameters.

The method introduced here has several advantages. In contrast to earlier hyperpolarization transfer techniques it does not require field-cycling and can be implemented using standard NMR equipment. As compared to field-cycling experiments where spin mixing at LAC regions can also be used to transfer spin hyperpolarization the method gives a further gain, coming from the fact that the RF-field is characterized by two parameters (amplitude and frequency) in contrast to the static field (a single parameter, field strength, is given). As a consequence, the method gives remarkable flexibility in choosing particular LACs to obtain the desired spin order. A possibility to perform both adiabatic and sudden switching of the RF-field increases this flexibility even further.

We anticipate that the method works for systems with a higher number of coupled spins and also for systems with lower symmetry. Although in these cases the analytical treatment is more complex, LAC positions can be predicted by theory as well as the resulting spin order coming from mixing at particular LACs. This strategy can be used to transfer hyperpolarization, CIDNP, PHIP or SABRE, to target spins suitable for NMR/MRI observation. Another potential application of our method is performing conversion between spin magnetization and long-lived spin order: by exploiting LACs in the rotating frame one can transfer magnetization into singletstate population, which often decays ${ }^{54,55}$ much slower than the usual longitudinal relaxation with time $T_{1}$. The validity of such an approach has been demonstrated by Franzoni et al., ${ }^{37}$ DeVience et $a .^{56}$ and also Warren et al. ${ }^{57,58}$ We anticipate that such a method of conversion between spin magnetization and long-lived spin order can further benefit from making experiments with variable parameters, $\nu_{\mathrm{rf}}$ and $\nu_{1}$, of the RF-field and different time profiles of switching the RF-field. 
As far as applications of the technique are concerned, it is highly promising for further development of the SABRE method. Since the SABRE-derived polarization of various substrates is efficiently formed only at low fields the technique in general is limited, since it requires either low-field NMR detection or fast field-cycling. However, to run SABRE experiments on modern high-field NMR spectrometers it is necessary to polarize the spins directly at high magnetic field. In this case the LAC-based polarization transfer methods can be a remedy. Our preliminary experiments show that such polarization transfer is operative making high-field SABRE feasible. These results are presented in a forthcoming publication.

Last but not least, bearing in mind the analogy with the classical Hartmann-Hahn cross-polarization technique, which is a standard method to polarize insensitive low- $\gamma$ nuclei, we anticipate that our developments are applicable also to heteronuclear NMR experiments with hyperpolarized spins. Notably, further progress in the methodology of LACs in the rotating frame can be exploited to transfer polarization from protons, which are primarily polarized in PHIP experiments, to other spin 1/2 nuclei. Since such nuclei usually have much longer relaxation times and lower thermal background signals, such studies are relevant for many applications of hyperpolarization in NMR spectroscopy and imaging.

\section{Acknowledgements}

This work was supported by the Russian Foundation for Basic Research (grants No. 13-03-00437 and 14-03-00397), grant of the President of the Russian Federation (MD-3279.2014.2), the Research Group Linkage Program of the Alexander von Humboldt Foundation, EU-COST Action TD1103, and the Ministry of Education and Science of the Russian Federation. We are thankful to Dr M. B. Franzoni for stimulating discussions.

\section{Notes and references}

1 K. H. Hausser and D. Stehlik, in Advances in Magnetic Resonance, ed. J. S. Waugh, Academic, New York, 1968, vol. 3, pp. 79-139.

2 T. Maly, G. T. Debelouchina, V. S. Bajaj, K.-N. Hu, C.-G. Joo, M. L. Mak-Jurkauskas, J. R. Sirigiri, P. C. A. van der Wel, J. Herzfeld, R. J. Temkin and R. G. Griffin, J. Chem. Phys., 2008, 128, 052211.

3 C. Griesinger, M. Bennati, H.-M. Vieth, C. Luchinat, G. Parigi, P. Hofer, F. Engelke, S. J. Glaser, V. Denysenkov and T. F. Prisner, Prog. Nucl. Magn. Reson. Spectrosc., 2012, 64, 4-28.

4 D. Stehlik and H.-M. Vieth, Mol. Cryst. Liq. Cryst., 1983, 93, 83-93.

5 D. Stehlik and H.-M. Vieth, in Pulsed Magn. Reson.: NMR, ESR, Opt., ed. D. M. S. Bagguley, Clarendon Press, Oxford, 1992, pp. 446-477.

6 K. M. Salikhov, Y. N. Molin, R. Z. Sagdeev and A. L. Buchachenko, Spin Polarization and Magnetic Effects in Chemical Reactions, Elsevier, Amsterdam, 1984.
7 W. Happer, Rev. Mod. Phys., 1972, 44, 169-249.

8 J. Natterer and J. Bargon, Prog. Nucl. Magn. Reson. Spectrosc., 1997, 31, 293-315.

9 R. A. Green, R. W. Adams, S. B. Duckett, R. E. Mewis, D. C. Williamson and G. G. R. Green, Prog. Nucl. Magn. Reson. Spectrosc., 2012, 67, 1-48.

10 J. Haupt, Phys. Lett., 1972, 38A, 389-390.

11 H. Jóhannesson, O. Axelsson and M. Karlsson, C. R. Phys., 2004, 5, 315-324.

12 T. Theis, P. Ganssle, G. Kervern, S. Knappe, J. Kitching, M. P. Ledbetter, D. Budker and A. Pines, Nat. Phys., 2011, 7, 571-575.

13 T. Theis, M. P. Ledbetter, G. Kervern, J. W. Blanchard, P. J. Ganssle, M. C. Butler, H. D. Shin, D. Budker and A. Pines, J. Am. Chem. Soc., 2012, 134, 3987-3990.

14 M. Lelli, D. Gajan, A. Lesage, M. A. Caporini, V. Vitzthum, P. Mieville, F. Heroguel, F. Rascon, A. Roussey, C. Thieuleux, M. Boualleg, L. Veyre, G. Bodenhausen, C. Coperet and L. Emsley, J. Am. Chem. Soc., 2011, 133, 2104-2107.

15 M. H. Lerche, S. Meier, P. R. Jensen, S. O. Hustvedt, M. Karlsson, J. O. Duus and J. H. Ardenkjaer-Larsen, NMR Biomed., 2011, 24, 96-103.

16 M. D. Lingwood and S. I. Han, in Annual Reports on NMR Spectroscopy, ed. G. A. Webb, Elsevier Academic Press, San Diego, 2011, vol. 73, pp. 83-126.

17 P. Berthault, G. Huber and H. Desvaux, Prog. Nucl. Magn. Reson. Spectrosc., 2009, 55, 35-60.

18 H. E. Moller, X. J. Chen, B. Saam, K. D. Hagspiel, G. A. Johnson, T. A. Altes, E. E. de Lange and H. U. Kauczor, Magn. Reson. Med., 2002, 47, 1029-1051.

19 D. Trease, V. S. Bajaj, J. Paulsen and A. Pines, Chem. Phys. Lett., 2011, 503, 187-190.

20 S. E. Day, M. I. Kettunen, F. A. Gallagher, D. E. Hu, M. Lerche, J. Wolber, K. Golman, J. H. Ardenkjaer-Larsen and K. M. Brindle, Nat. Med., 2007, 13, 1382-1387.

21 L. Frydman and D. Blazina, Nat. Phys., 2007, 3, 415-419.

22 V. S. Bajaj, M. L. Mak-Jurkauskas, M. Belenky, J. Herzfeld and R. G. Griffin, Proc. Natl. Acad. Sci. U. S. A., 2009, 106, 9244-9249.

23 M. S. Albert, G. D. Cates, B. Driehuys, W. Happer, B. Saam, C. S. Springer and A. Wishnia, Nature, 1994, 370, 199-201.

24 L.-S. Bouchard, S. R. Burt, M. S. Anwar, K. V. Kovtunov, I. V. Koptyug and A. Pines, Science, 2008, 319, 442-445.

25 L. Schröder, T. J. Lowery, C. Hilty, D. E. Wemmer and A. Pines, Science, 2006, 314, 446-449.

26 J. H. Lee, Y. Okuno and S. Cavagnero, J. Magn. Reson., 2014, 241, 18-31.

27 R. W. Adams, J. A. Aguilar, K. D. Atkinson, M. J. Cowley, P. I. Elliott, S. B. Duckett, G. G. Green, I. G. Khazal, J. LopezSerrano and D. C. Williamson, Science, 2009, 323, 1708-1711.

28 K. L. Ivanov, K. Miesel, A. V. Yurkovskaya, S. E. Korchak, A. S. Kiryutin and H.-M. Vieth, Appl. Magn. Reson., 2006, 30, 513-534.

29 K. L. Ivanov, A. V. Yurkovskaya and H.-M. Vieth, J. Chem. Phys., 2008, 128, 154701. 
30 K. Miesel, PhD thesis, Free University of Berlin, 2008.

31 A. S. Kiryutin, A. V. Yurkovskaya, R. Kaptein, H.-M. Vieth and K. L. Ivanov, J. Phys. Chem. Lett., 2013, 4, 2514-2519.

32 A. N. Pravdivtsev, A. V. Yurkovskaya, R. Kaptein, K. Miesel, H.-M. Vieth and K. L. Ivanov, Phys. Chem. Chem. Phys., 2013, 15, 14660-14669.

33 A. N. Pravdivtsev, A. V. Yurkovskaya, H.-M. Vieth and K. L. Ivanov, J. Chem. Phys., 2013, 139, 046348.

34 A. N. Pravdivtsev, A. V. Yurkovskaya, H.-M. Vieth, K. L. Ivanov and R. Kaptein, ChemPhysChem, 2013, 14, 3327-3331.

35 L. Buljubasich, M. B. Franzoni, H. W. Spiess and K. Münnemann, J. Magn. Reson., 2012, 219, 33.

36 M. B. Franzoni, L. Buljubasich, H.-W. Spiess and K. Münnemann, J. Am. Chem. Soc., 2012, 134, 10393-10396.

37 M. B. Franzoni, D. Graafen, L. Buljubasich, L. M. Schreiber, H. W. Spiess and K. Münnemann, Phys. Chem. Chem. Phys., 2013, 15, 17233-17239.

38 M. Benkert and H.-M. Vieth, Chem. Phys. Lett., 1994, 230, 153-159.

39 A. Henstra and W. T. Wenckebach, Mol. Phys., 2008, 106, 859.

40 T. R. Eichhorn, M. Haag, B. van den Brandt, P. Hautle and W. T. Wenckebach, Chem. Phys. Lett., 2013, 555, 296-299.

41 A. S. Kiryutin, K. L. Ivanov, A. V. Yurkovskaya, R. Kaptein and H.-M. Vieth, Z. Phys. Chem., 2012, 226, 1343-1362.

42 A. S. Kiryutin, K. L. Ivanov, A. V. Yurkovskaya, H.-M. Vieth and N. N. Lukzen, Phys. Chem. Chem. Phys., 2013, 15, 14248-14255.
43 E. A. Nasibulov, A. N. Pravdivtsev, A. V. Yurkovskaya, N. N. Lukzen, H.-M. Vieth and K. L. Ivanov, Z. Phys. Chem., 2013, 227, 929-953.

44 J. von Neumann and E. Wigner, Phys. Z., 1929, 30, 467-470. 45 L. D. Landau and E. M. Lifshitz, Quantum Mechanics, Butterworth-Heinemann, Oxford, 3rd edn, 1991.

46 S. R. Hartmann and E. L. Hahn, Phys. Rev., 1962, 128, 2042-2053.

47 S. Hediger, B. H. Meier, N. D. Kurur, G. Bodenhausen and R. R. Ernst, Chem. Phys. Lett., 1994, 223, 283-288.

48 P. Pelupessy and E. Chiarparin, Concepts Magn. Reson., 2000, 12, 103-124.

49 W. R. Janzen, J. Magn. Reson., 1973, 12, 71-78.

50 E. Chiarparin, P. Pelupessy and G. Bodenhausen, Mol. Phys., 1998, 95, 759-767.

51 R. Verel and B. H. Meier, ChemPhysChem, 2004, 5, 851-862. 52 V. S. Malinovsky and J. T. Krause, Eur. Phys. J. D, 2001, 14, 147-155.

53 N. N. Lukzen and U. E. Steiner, Mol. Phys., 1995, 86, 1271-1282.

54 G. Pileio, Prog. Nucl. Magn. Reson. Spectrosc., 2010, 56, 217-231.

55 M. H. Levitt, Annu. Rev. Phys. Chem., 2012, 63, 89-105.

56 S. J. DeVience, R. L. Walsworth and M. S. Rosen, Phys. Rev. Lett., 2013, 111, 173002.

57 Y. Feng, R. M. Davis and W. S. Warren, Nat. Phys., 2012, 8, 831-837.

58 K. Claytor, T. Theis, Y. Feng and W. Warren, J. Magn. Reson., 2014, 239, 81-86. 